\title{
INTERVAL-VALUED SMOOTH TOPOLOGICAL SPACES
}

\author{
JeOng YeOl $\mathrm{ChOI}^{1}$, So Ra Kim ${ }^{2}$ and Kul HuR ${ }^{3}$
}

\begin{abstract}
We list two kinds of gradation of openness and we study in the sense of the followings:

(i) We give the definition of IVGO of fuzzy sets and obtain some basic results.

(ii) We give the definition of interval-valued gradation of clopeness and obtain some properties.

(iii) We give the definition of a subspace of an interval-valued smooth topological space and obtain some properties.

(iv) We investigate some properties of gradation preserving (in short, IVGP) mappings.
\end{abstract}

\section{Introduction}

In 1965, Zadeh [19] introduced the concept of fuzzy sets as a generalization of (ordinary) subsets. Soon after, Chang [6] was the first to introduce the notion of a fuzzy topology $T$ on a set $X$ by axiomatizing a collection $T$ of fuzzy sets in $X$ as follows:

(i) $\emptyset, X \in T$,

(ii) $A, B \in T \Rightarrow A \cap B \in T$,

(iii) $\left\{A_{\alpha}\right\}_{\alpha \in \Gamma} \subset T \Rightarrow \bigcup_{\alpha \in \Gamma} A_{\alpha} \in T$,

where he reffered to each member of $T$ as an open set.

Some authors $[7,9,10,18]$ noted that fuzziness in it was absent, and Sostak[18] began the study of fuzzy structures of the topological type and called a function $\tau: I^{X} \rightarrow I$, satisfying the following conditions:

(i) $\tau(\emptyset)=\tau(X)=1$,

(ii) $\tau(A \cap B) \geq \tau(A) \wedge \tau(B), \forall A, B \in I^{X}$,

Received June 08, 2010. Accepted November 22, 2010.

${ }^{3}$ Corresponding author.

2000 Mathematics Subject Classification : 54A40.

Keywords and phrases. t-norm : interval-valued gradation of openness (resp. closedness and clopenness), interval-valued preserving mapping.

${ }^{1}$ This paper was supported by Wonkwang University in 2009. 
(iii) $\tau\left(\bigcup_{\alpha \in \Gamma} A_{\alpha}\right) \geq \bigwedge_{\alpha \in \Gamma} \tau\left(A_{\alpha}\right), \forall\left\{A_{\alpha}\right\}_{\alpha \in \Gamma} \subset I^{X}$,

as a fuzzy topology on $X$. In this case, the pair $(X, \tau)$ was called a fuzzy topological space (in short, FTS) and $\tau(A)$ was called the degree of openness of the fuzzy set $A$.

On the other hand, various generalizations of the notion of fuzzy set have been done by many authors. Zadeh[20] introduced the idea of interval-valued fuzzy sets. Later, Atanassov[1] introduced the concept of intuitionistic fuzzy set. Moreover, Atanassov and Gargov[2] introduced the notion of interval-valued intuitionistic fuzzy sets as the generalization of both interval-valued fuzzy sets and intuitionistic fuzzy sets. Some researchers $[1,2,3,4,5]$ have worked mainly on operators and relations on intuitionistic fuzzy sets and interval-valued intuitionistic fuzzy sets. Çoker[8] introduced the idea of the topology of intuitionistic fuzzy sets, and Hur et.al[11,12] investigated some properties of intuitionistic fuzzy topological groups and intuitionistic fuzzy topological spaces. Samanta and Mondal[16,17] introduced the definitions of the topology of intervalvalued fuzzy sets and the topology of interval-valued intuitionistic fuzzy sets, respectively. In particular, recently, Mondal and Samanta[14,15] introduced the notion of intuitionistic gradation of openness.

In this pater, we list two kinds of gradation of openness and we the sense of the followings:

(i) We give the definition of IVGO of fuzzy sets and obtain some basic results.

(ii) We give the definition of interval-valued gradation of clopeness and obtain some properties.

(iii) We give the definition of a subspace of an interval-valued smooth topological space and obtain some properties.

(iv) We investigate some properties of gradation preserving (In short, IVGP) mappings.

\section{Preliminaries}

Throughout this paper, $X$ will denote a nonempty set; $I=[0,1]$, the closed unit interval of the real line; $I_{0}=(0,1] ; I_{1}=[0,1) ; I^{X}=$ the set of all fuzzy sets in $X$. In particular, $\emptyset$ and $X$ denote the empty fuzzy set and the whole fuzzy set in $X$ defined by $\emptyset(x)=0$ and $X(x)=1, \forall x \in X$, respectively. All other notations are standard notations of fuzzy set theory. A complex mapping $A=\left(\mu_{A}, \nu_{A}\right): X \rightarrow I \times I$ satisfying 
the condition $\mu_{A}(x)+\nu_{A}(x) \leq 1, \forall x \in X$, is called an intuitionistic fuzzy set in $X$, and $0_{\sim}$ and $1_{\sim}$ denote the empty intuitionistic fuzzy set and the whole intuitionistic fuzzy set in $X$ defined by $0_{\sim}(x)=(0,1)$ and $1_{\sim}(x)=(1,0), \forall x \in X$, respectively. We will denote the set of all intuitionistic fuzzy sets in $X$ as $\operatorname{IFS}(X)$. Also all the notations are standard notations of intuitionistic fuzzy set theory.

Let $D(I)$ be the set of all closed subintervals of the unit interval $I$. The elements of $D(I)$ are generally denoted by capital letters $M, N, \ldots$, and note that $M=\left[M^{L}, M^{U}\right]$, where $M^{L}$ and $M^{U}$ are the lower and upper points respectively. Especially, we denote $\boldsymbol{a}=[a, a]$ for every $a \in(0,1)$. We also note that

(i) $(\forall M, N \in D(I))\left(M=N \Leftrightarrow M^{L}=N^{L}, M^{U}=N^{U}\right)$.

(ii) $(\forall M, N \in D(I))\left(M \leq N \Leftrightarrow M^{L} \leq N^{L}, M^{U} \leq N^{U}\right)$.

For every $M \in D(I)$, the complement of $M$, denoted by $M^{c}$, is defined by $M^{c}=1-M=\left[1-M^{U}, 1-M^{L}\right]$.

Definition 2.1[20]. Let $X$ be a given nonempty set. A mapping $A=\left[A^{L}, A^{U}\right]: X \rightarrow D(I)$ is called an interval valued fuzzy set (briefly, IVFS) in $X$, where $A^{L}$ and $A^{U}$ are fuzzy sets in $X$ satisfying $A^{L}(x) \leq A^{U}(x)$ and $A(x)=\left[A^{L}(x), A^{U}(x)\right]$ for each $x \in X$, and $A^{L}(x)$ and $A^{U}(x)$ are called the lower and upper end points of $A(x)$, respectively

It is clear that every fuzzy set $A$ in $X$ is an IVFS of the form $A=[A, A]$. For any $[a, b] \in D(I)$, the IVFS whose value is the interval $[a, b]$ for all $x \in X$ is denoted by $\widetilde{[a, b]}$, i.e., $\widetilde{[a, b}](x)=[a, b]$ for each $x \in X$. For any $a \in I$, the IVFS whose value is $\boldsymbol{a}$ for all $x \in X$ is denoted by simply $\widetilde{a}$, i.e., $\widetilde{a}(x)=\boldsymbol{a}$ for each $x \in X$. $\widetilde{0}$ and $\widetilde{1}$ denote the empty interral-valued fuzzy set and the whole interval-valued fuzzy set in $X$, respectively. For a point $p \in X$ and for $[a, b] \in D(I)$ with $b>0$, the IVFS which takes the value $[a, b] \in D(I)$ at $p$ and $\mathbf{0}$ elsewhere in $X$ is called an interval-valued fuzzy point(briefly, an IVFP) and is denoted by $p_{[a, b]}$. In particular, if $b=a$, it is also denoted by $p_{\boldsymbol{a}}$. We will denote by $D(I)^{X}$ and $\operatorname{IVF}_{P}(X)$ the set of all $\operatorname{IVFS}_{S}$ and the set of all IVF points in $X$ by $D(I)^{X}$ and $\operatorname{IVF}_{P}(X)$, respectively.

Notation. Let $X=\left\{x_{1}, x_{2}, \ldots, x_{n}\right\}$. Then $A=\left(\left[a_{1}, b_{1}\right],\left[a_{2}, b_{2}\right], \ldots,\left[a_{n}, b_{n}\right]\right)$ denotes an IVFS in $X$ such that $A^{L}\left(x_{i}\right)=a_{i}$ and $A^{U}\left(x_{i}\right)=b_{i}$, for all $i=1,2, \ldots, n$. 
Definition 2.2[16]. Let $A, B \in D(I)^{X}$. Then:

(a) $A \subset B$ iff $A^{L}(x) \leq B^{L}(x)$ and $A^{U}(x) \leq B^{U}(x)$ for all $x \in X$.

(b) $A=B$ iff $A \subset B$ and $B \subset A$.

(c) The complement $A^{c}$ of $A$ is defined by $A^{c}=\left[1-A^{U}(x), 1-A^{L}(x)\right]$ for all $x \in X$.

(d) If $\left\{A_{\alpha}: \alpha \in \Gamma\right\}$ is an arbitrary subset of $D(I)^{X}$, then

$$
\begin{aligned}
& \bigcap A_{\alpha}(x)=\left[\bigwedge_{\alpha \in \Gamma} A_{\alpha}^{L}(x), \bigwedge_{\alpha \in \Gamma} A_{\alpha}^{U}(x)\right], \\
& \bigcup A_{\alpha}(x)=\left[\bigvee_{\alpha \in \Gamma} A_{\alpha}^{L}(x), \bigvee_{\alpha \in \Gamma} A_{\alpha}^{U}(x)\right] .
\end{aligned}
$$

Definition 2.3[16]. Let $T \subset D(I)^{X}$. Then $T$ is called an intervalvalued fuzzy topology(in short, IVFT) on $X$ if it satisfies the following conditions:

(i) $\widetilde{0}, \widetilde{1} \in T$,

(ii) $A, B \in T \Rightarrow A \cap B \in T$,

(iii) $\left\{A_{\alpha}\right\}_{\alpha \in \Gamma} \subset T \Rightarrow \bigcup_{\alpha \in \Gamma} A_{\alpha} \in T$.

In this case, each member of $T$ is called an IVF open set and the pair $(X, T)$ is called an interval-valued fuzzy topological space(in short, IVFTS $). A \in D(I)^{X}$ is called closed in $(X, T)$ if $A^{c} \in T$.

As in ordinary topologies, the indiscrete topology of IVF sets contains only $\widetilde{1}$ and $\widetilde{0}$, while the discrete topology of IVF sets contains all IVF sets. These two topologies are denoted by $T^{0}$ and $T^{1}$, respectively.

\section{Interval-valued gradation of openness}

Definition 3.1[7,18]. A mapping $\tau: I^{X} \rightarrow I$ is called a gradation of openness (in short, $G O$ ) or a smooth topology on $X$ if it satisfies the following conditions:

$$
\begin{aligned}
& \text { (GO1) } \tau(\emptyset)=\tau(X)=1, \\
& \text { (GO2) } \tau(A) \geq r \text { and } \tau(B) \geq r \Rightarrow \tau(A \cap B) \geq r, \text { for any } A, B \in I^{X}, \\
& \text { (GO3) } \tau\left(A_{\alpha}\right) \geq r, \forall \alpha \in \Gamma \Rightarrow \tau\left(\bigcup_{\alpha \in \Gamma} A_{\alpha}\right) \geq r, \text { for any }\left\{A_{\alpha}\right\}_{\alpha \in \Gamma} \subset I^{X},
\end{aligned}
$$

where $r \in I_{0}$; or equivalently:

$$
\begin{aligned}
& (\mathrm{GO} 1)^{\prime} \tau(\emptyset)=\tau(X)=1, \\
& (\mathrm{GO} 2)^{\prime} \tau(A \cap B) \geq \tau(A) \wedge \tau(B), \text { for any } A, B \in I^{X},
\end{aligned}
$$


$(\mathrm{GO} 3)^{\prime} \tau\left(\bigcup_{\alpha \in \Gamma} A_{\alpha}\right) \geq \bigwedge_{\alpha \in \Gamma} \tau\left(A_{\alpha}\right)$, for any $\left\{A_{\alpha}\right\}_{\alpha \in \Gamma} \subset I^{X}$.

The pair $(X, \tau)$ is called a smooth topological space(in short, STS).

Definition 3.2[14]. A complex mapping $\tau=\left(\mu_{\tau}, \nu_{\tau}\right): I^{X} \rightarrow I \times$ $I$ is called an intuitionistic gradation of openness(in short, IGO) an intuitionistic smooth topology on $X$ if it satisfies the following conditions:

(IGO1) $\mu_{\tau}(A)+\nu_{\tau}(A) \leq 1$, for each $A \in I^{X}$,

(IGO2) $\tau(\emptyset)=\tau(X)=(1,0)$,

(IGO3) $\mu_{\tau}(A \cap B) \geq \mu_{\tau}(A) \wedge \mu_{\tau}(B)$ and $\nu_{\tau}(A \cap B) \leq \nu_{\tau}(A) \vee \nu_{\tau}(B)$, for any $A, B \in I^{X}$,

(IGO4) $\mu_{\tau}\left(\bigcup_{\alpha \in \Gamma} A_{\alpha}\right) \geq \bigwedge_{\alpha \in \Gamma} \mu_{\tau}\left(A_{\alpha}\right)$ and $\nu_{\tau}\left(\bigcup_{\alpha \in \Gamma} A_{\alpha}\right) \leq \bigvee_{\alpha \in \Gamma} \nu_{\tau}\left(A_{\alpha}\right)$, for any $\left\{A_{\alpha}\right\}_{\alpha \in \Gamma} \subset I^{X}$.

The triple $\left(X, \mu_{\tau}, \nu_{\tau}\right)$ is called an intuitionistic smooth topological space(in short, ISTS), and $\mu_{\tau}$ and $\nu_{\tau}$ may be interpreted as gradation of openness and nonopenness, respectively.

Definition 3.3. A mapping $\tau=\left[\tau^{L}, \tau^{U}\right]: I^{X} \rightarrow D(I)$ is called an interval-valued gradation of openess(in short, IVGO) or an intervalvalued smooth topology on $X$ if it satisfies the following conditions:

(IVGO1) $\tau^{L}(A) \leq \tau^{U}(A)$, for each $A \in I^{X}$,

(IVGO2) $\tau(\emptyset)=\tau(X)=\mathbf{1}$,

$(\mathrm{IVGO} 3) \tau^{L}(A \cap B) \geq \tau^{L}(A) \wedge \tau^{L}(B)$ and $\tau^{U}(A \cap B) \geq \tau^{U}(A) \wedge \tau^{U}(B)$, for any $A, B \in I^{X}$,

$\left(\right.$ IVGO4) $\tau^{L}\left(\bigcup_{\alpha \in \Gamma} A_{\alpha}\right) \geq \bigwedge_{\alpha \in \Gamma} \tau^{L}\left(A_{\alpha}\right)$ and $\tau^{U}\left(\bigcup_{\alpha \in \Gamma} A_{\alpha}\right) \geq \bigwedge_{\alpha \in \Gamma} \tau^{U}\left(A_{\alpha}\right)$, for any $\left\{A_{\alpha}\right\}_{\alpha \in \Gamma} \subset I^{X}$.

The pair $(X, \tau)$ is called an interval-valued smooth topological space(in short, IVSTS).

We will denote the set of all $\mathrm{GO} s[$ resp. IGO $s$ and IVGO $s]$ on $X$ as $\mathrm{GO}(X)[\operatorname{resp}, \operatorname{IGO}(X)$ and $\operatorname{IVGO}(X)]$.

Example 3.3. (a) Let $T$ be the topology on $\mathbb{R}$ generated by $\mathcal{B}=\{(a, b]$ : $a, b \in \mathbb{R}$ and $a<b\}$ as a subbase, and let $T_{o}$ be the family of all open sets in $\mathbb{R}$ with respect to (in short, w.r.t.) the usual topology on $\mathbb{R}$, where $\mathbb{R}$ denotes the set of all real numbers. We define the mapping $\tau=\left[\tau^{L}, \tau^{U}\right]: I^{\mathbb{R}} \rightarrow D(I)$ as follows: For each $A \in I^{X}$, 


$$
\tau(A)=\left\{\begin{array}{lc}
\mathbf{1} & \text { if } A \in T_{o}, \\
{[0.5,0.7]} & \text { if } A \in T \backslash T_{o}, \\
\mathbf{0} & \text { otherwise. }
\end{array}\right.
$$

Then it can easily seen that $\tau \in \operatorname{IVGO}(X)$.

(b) Let $a<b$ in $\mathbb{R}$ and let $\lambda \in I_{o}$. We define the mapping $A: \mathbb{R} \rightarrow I$ as follows: For each $x \in \mathbb{R}$,

$$
A(x)=\left\{\begin{array}{cc}
1 & \text { if } x \in(a, b), \\
\lambda & \text { if } x=b, \\
0 & \text { othewise. }
\end{array}\right.
$$

Then clearly $A \in I^{X}$ and we write $A=(a, b)_{\lambda}$. Let $\mathcal{B}=\left\{(a, b)_{\lambda}: a, b \in\right.$ $\mathbb{R}, a<b$ and $\left.\lambda \in I_{o}\right\}$, let $T$ be the chang's fuzzy topology generated by $\mathcal{B}$ as a subbase and let $T_{o}=\left\{\chi_{o}: O\right.$ is an open set in $\left.\mathbb{R}\right\}$. Any $A \in T \backslash T_{o}$ can be expressed as

$$
A=\bigcup_{\alpha \in \Gamma} A_{\alpha}(3.1)
$$

where $A_{\alpha}=\left(a_{\alpha}, b_{\alpha}\right)_{\lambda}$ and $\Gamma$ is countable. We define the mapping $\tau=$ $\left[\tau^{L}, \tau^{U}\right]: I^{\mathbb{R}} \rightarrow D(I)$ as follows: For each $A \in I^{X}$,

$\tau(A)=\left\{\begin{array}{l}\mathbf{1} \text { if } A \in T_{o} \\ {[1-0.5 \lambda, 0.7 \lambda] \text { if } A=(a, b)_{\lambda}} \\ {\left[\bigwedge_{\alpha \in \Gamma} \tau^{L}\left(A_{\alpha}\right), \bigwedge_{\alpha \in \Gamma} \tau^{U}\left(A_{\alpha}\right)\right] \text { if } A \text { is expressed in the form (3.1) }} \\ \mathbf{0} \text { otherwise. }\end{array}\right.$

Then we can easily see that $\tau \in \operatorname{IVGO}(X)$.

The following is the immediate result of Definitions 3.1, 3.2 and 3.3.

Proposition 3.4. (a) If $\tau \in \operatorname{GO}(X)$, then $\left(\tau, \tau^{c}\right) \in \operatorname{IGO}(X)$ and $\tau=$ $[\tau, \tau] \in \operatorname{IVGO}(X)$, where $\tau^{c}(A)=1-\tau(A), \forall A \in I^{X}$.

(b) If $\tau \in \operatorname{IGO}(X)$ [resp. $\operatorname{IVGO}(X)]$, then $\mu_{\tau}, \nu_{\tau}^{c} \in \mathrm{GO}(X)$ [resp. $\left.\tau^{L}, \tau^{U} \in \mathrm{GO}(X)\right]$.

Proposition 3.5. We define two mappings $f: \operatorname{IVGO}(X) \rightarrow \operatorname{IGO}(X)$ and $g: \operatorname{IGO}(X) \rightarrow \operatorname{IVGO}(X)$ as follows, respectively:

and

$$
f(\tau)=f\left(\left[\tau^{L}, \tau^{U}\right]\right)=\left(\tau^{L},\left(\tau^{U}\right)^{c}\right), \forall \tau \in \operatorname{IVGO}(X)
$$

$g(\tau)=g\left(\left(\mu_{\tau}, \nu_{\tau}\right)\right)=\left[\mu_{\tau}, \nu_{\tau}^{c}\right], \forall \tau \in \operatorname{IGO}(X)$. 
Then $g \circ f=1_{\mathrm{IVGO}(\mathrm{X})}$ and $f \circ g=1_{\mathrm{IGO}(\mathrm{X})}$.

Proof. It can be easily seen that $f$ and $g$ are functions. Let $\tau \in$ $\operatorname{IVGO}(X)$. Then

$$
\begin{aligned}
& g \circ f(\tau)=g\left(\left(\tau^{L},\left(\tau^{U}\right)^{c}\right)\right) \\
& =\left[\tau^{L},\left(\left(\tau^{U}\right)^{c}\right)^{c}\right] \\
& =\left[\tau^{L}, \tau^{U}\right]=\tau=1_{\mathrm{IVGO}(\mathrm{X})} \\
& \text { Now let } \tau \in \operatorname{IGO}(X) . \text { Then } \\
& \quad f \circ g(\tau)=f\left(\left[\mu_{\tau},\left(\nu_{\tau}\right)^{c}\right]\right) \\
& =\left(\mu_{\tau},\left(\left(\nu_{\tau}\right)^{c}\right)^{c}\right) \\
& =\left(\mu_{\tau}, \nu_{\tau}\right)=\tau=1_{\mathrm{IGO}(\mathrm{X}) .}
\end{aligned}
$$

This completes the proof.

Remark 3.5. Proposition 3.5 shows the concepts of IVGO and IGO to be equipollent generalizations of one of GO.

Definition 3.6[7]. A mapping $\mathrm{F}: I^{X} \rightarrow I$ is called a gradation of closedness(in short, $G C$ ) or a smooth cotopology on $X$ if it satisfies the following conditions:

(GC1) $\mathcal{F}(\emptyset)=\mathcal{F}(X)=1$,

(GC2) $\mathcal{F}(A) \geq r$ and $\mathcal{F}(B) \geq r \Rightarrow \mathcal{F}(A \cup B) \geq r$, for any $A, B \in I^{X}$,

(GC3) $\mathcal{F}\left(A_{\alpha}\right) \geq r, \forall \alpha \in \Gamma \Rightarrow \mathcal{F}\left(\bigcap_{\alpha \in \Gamma} A_{\alpha}\right) \geq r$, for any $\left\{A_{\alpha}\right\} \subset I^{X}$,

where $r \in I_{o}$; or equivalently:

$(\mathrm{GC} 1)^{\prime} \mathcal{F}(\emptyset)=\mathcal{F}(X)=1$,

$(\mathrm{GC} 2)^{\prime} \mathcal{F}(A \cup B) \geq \mathcal{F}(A) \cap \mathcal{F}(B)$, for any $A, B \in I^{X}$,

$(\mathrm{GC} 3)^{\prime} \mathcal{F}\left(\bigcap_{\alpha \in \Gamma} A_{\alpha}\right) \geq \bigwedge_{\alpha \in \Gamma} \mathcal{F}\left(A_{\alpha}\right)$, for any $\left\{A_{\alpha}\right\}_{\alpha \in \Gamma} \subset I^{X}$.

Definition 3.7[14]. A complex mapping $\mathcal{F}=\left(\mu_{\mathcal{F}}, \nu_{\mathcal{F}}\right): I^{X} \rightarrow I \times I$ is called an intuitionistic gradation of closedness(in short, IGC) an intuitionistic smooth cotopology on $X$ if it satisfies the following conditions:

(IGC1) $\mu_{\mathcal{F}}(A)+\nu_{\mathcal{F}}(A) \leq 1$, for each $A \in I^{X}$,

(IGC2) $\mathcal{F}(\emptyset)=\mathcal{F}(X)=(1,0)$,

$(\mathrm{IGC} 3) \mu_{\mathcal{F}}(A \cup B) \geq \mu_{\mathcal{F}}(A) \wedge \mu_{\mathcal{F}}(B)$ and $\nu_{\mathcal{F}}(A \cup B) \leq \nu_{\mathcal{F}}(A) \vee \nu_{\mathcal{F}}(B)$, for any $A, B \in I^{X}$.

(IGC4) $\mu_{\mathcal{F}}\left(\bigcap_{\alpha \in \Gamma} A_{\alpha}\right) \geq \bigwedge_{\alpha \in \Gamma} \mu_{\mathcal{F}}\left(A_{\alpha}\right)$ and $\nu_{\mathcal{F}}\left(\bigcap_{\alpha \in \Gamma} A_{\alpha}\right) \leq \bigvee_{\alpha \in \Gamma} \nu_{\mathcal{F}}\left(A_{\alpha}\right)$, for any $\left\{A_{\alpha}\right\}_{\alpha \in \Gamma} \subset I^{X}$. 
Definition 3.8. A mapping $\mathcal{F}=\left[\mathcal{F}^{L}, \mathcal{F}^{U}\right]: I^{X} \rightarrow D(I)$ is called an interval-valued gradation of closedness(in short, IVGC) an intervalvalued smooth cotopology on $X$ if it satisfies the following conditions:

(IVGC1) $\mathcal{F}^{L}(A) \leq \mathcal{F}^{U}(A)$, for each $A \in I^{X}$,

$($ IVGC2) $\mathcal{F}(\emptyset)=\mathcal{F}(X)=1$,

$\left(\right.$ IVGC3) $\mathcal{F}^{L}(A \cup B) \geq \mathcal{F}^{L}(A) \wedge \mathcal{F}^{L}(B)$ and $\mathcal{F}^{U}(A \cup B) \geq \mathcal{F}^{U}(A) \wedge$ $\mathcal{F}^{U}(B)$, for any $A, B \in I^{X}$,

$\left(\right.$ IVGC4) $\mathcal{F}^{L}\left(\bigcap_{\alpha \in \Gamma} A_{\alpha}\right) \geq \bigwedge_{\alpha \in \Gamma} \mathcal{F}^{L}\left(A_{\alpha}\right)$ and $\mathcal{F}^{U}\left(\bigcap_{\alpha \in \Gamma} A_{\alpha}\right) \geq \bigwedge_{\alpha \in \Gamma} \mathcal{F}^{U}\left(A_{\alpha}\right)$, for any $\left\{A_{\alpha}\right\}_{\alpha \in \Gamma} \subset I^{X}$.

We will denote the set of all GCs[resp. IGCs and IVGCs] an $X$ as $\mathrm{GC}(X)[$ resp. $\operatorname{IGC}(X)$ and $\operatorname{IVGC}(X)]$.

The following is the generalization of Propositions 2.3, 2.4 and Corollary 2.5 in[7], as well as the analogue to Theorem 2.6 in[14].

Proposition 3.9. (a) For each $\tau \in \operatorname{IVGO}(X)$, we define the mapping $\mathcal{F}_{\tau}: I^{X} \rightarrow D(I)$ as follows: For each $A \in I^{X}$,

$\mathcal{F}_{\tau}(A)=\tau\left(A^{c}\right)$.

Then $\mathcal{F}_{\tau} \in \operatorname{IVGC}(X)$.

(b) For each $\mathcal{F} \in \operatorname{IVGC}(X)$, we define the mapping $\tau_{\mathcal{F}}: I^{X} \rightarrow D(I)$ as follows: For each $A \in I^{X}$,

$\tau_{\mathcal{F}}(A)=\mathcal{F}\left(A^{c}\right)$.

Then $\tau_{\mathcal{F}} \in \operatorname{IVGO}(X)$.

(c) $\tau_{\mathcal{F}_{\tau}}=\tau$ and $\mathcal{F}_{\tau_{\mathcal{F}}}=\mathcal{F}$.

Proof. (a) It is clear that $\mathcal{F}_{\tau}$ satisfies the conditions (IVGC1) and (IVGC2). Let $A, B \in I^{X}$. Then

$\mathcal{F}_{\tau}^{L}(A \cup B)=\tau^{L}\left((A \cup B)^{c}\right)=\tau^{L}\left(A^{c} \cap B^{c}\right)$

$\geq \tau^{L}\left(A^{c}\right) \wedge \tau^{L}\left(B^{c}\right)$ [By the condition (IVGO3)]

$=\mathcal{F}_{\tau}^{L}(A) \wedge \mathcal{F}_{\tau}^{L}(B)$. [By the definition of $\left.\mathcal{F}_{\tau}\right]$

Similarly, we have $\mathcal{F}_{\tau}^{U}(A)(A \cup B) \geq \mathcal{F}_{\tau}^{U}(A) \wedge \mathcal{F}_{\tau}^{U}(B)$. Thus $\mathcal{F}_{\tau}$ satisfies the condition (IVGC3). Now let $\left\{A_{\alpha}\right\}_{\alpha \in \Gamma} \subset I^{X}$. Then

$\mathcal{F}_{\tau}^{L}\left(\bigcap_{\alpha \in \Gamma} A_{\alpha}\right)=\tau^{L}\left(\left(\bigcap_{\alpha \in \Gamma} A_{\alpha}\right)^{c}\right)=\tau^{L}\left(\bigcup_{\alpha \in \Gamma} A_{\alpha}^{c}\right)$

$\geq \bigwedge_{\alpha \in \Gamma} \tau^{L}\left(A_{\alpha}^{c}\right)$ [By the condition (IVGO4)]

$=\bigwedge_{\alpha \in \Gamma} \mathcal{F}_{\tau}^{L}\left(A_{\alpha}^{c}\right),\left[\right.$ By the definition of $\left.\mathcal{F}_{\tau}\right]$ 
Similarly, we have $\mathcal{F}_{\tau}^{L}\left(\bigcap_{\alpha \in \Gamma} A_{\alpha}\right) \geq \bigwedge_{\alpha \in \Gamma} \mathcal{F}_{\tau}^{L}\left(A_{\alpha}\right)$. So $\mathcal{F}_{\tau}$ satisfies the condition (IVGC4). Hence $\mathcal{F}_{\tau} \in \operatorname{IVGC}(X)$.

The proof of (b) is similar to one of (a) and (c) are the immediate results of the definitions of $\mathcal{F}_{\tau}$ and $\tau_{\mathcal{F}}$.

Definition 3.10. Let $\left\{\tau_{\alpha}\right\}_{\alpha \in \Gamma} \subset \operatorname{IVGO}(X)$. Then the intersection of $\left\{\tau_{\alpha}\right\}_{\alpha \in \Gamma}$, denoted by $\bigcap_{\alpha \in \Gamma} \tau_{\alpha}$, is defined as follows: For each $A \in I^{X}$,

$$
\left(\bigcap_{\alpha \in \Gamma} \tau_{\alpha}\right)(A)=\left[\bigwedge_{\alpha \in \Gamma} \tau_{\alpha}^{L}(A), \bigwedge_{\alpha \in \Gamma} \tau_{\alpha}^{U}(A)\right]
$$

The following is the immediate result of Definitions 3.3 and 3.10 .

Proposition 3.11. Let $\left\{\tau_{\alpha}\right\}_{\alpha \in \Gamma} \subset \operatorname{IVGO}(X)$. Then $\bigcap_{\alpha \in \Gamma} \tau_{\alpha} \in \operatorname{IVGO}(X)$.

Definition 3.12. We define a relation " $\leq$ " on $\operatorname{IVGO}(X)$ as follows: $\tau \leq \eta \Leftrightarrow \tau^{L} \leq \eta^{L}$ and $\tau^{U} \leq \eta^{U}$, for any $\tau, \eta \in \operatorname{IVGO}(X)$.

It can be easily seen that $(\operatorname{IVGO}(X), \leq)$ is a partially ordered set.

Remark 3.13. We define two mappings $\tau_{\mathbf{0}}, \tau_{\mathbf{1}}: I^{X} \rightarrow D(I)$ as follows: For each $A \in I^{X}$,

$$
\tau_{\mathbf{0}}(A)=\left\{\begin{array}{l}
\mathbf{1} \text { if } A=\varnothing \text { or } A=X \\
\mathbf{0} \text { if } A \in I^{X} \backslash\{\varnothing, X\}
\end{array}\right.
$$

and

$$
\tau_{\mathbf{1}}(A)=\mathbf{1}
$$

Then we can easily see that $\tau_{\mathbf{0}}, \tau_{\mathbf{1}} \in \operatorname{IVGO}(X)$ and $\tau_{\mathbf{0}} \leq \tau \leq \tau_{\mathbf{1}}, \forall \tau \in$ $\operatorname{IVGO}(X)$.

The followings is the immediate result of Proposition 3.11 and Remark 3.13.

Proposition 3.14. $(\operatorname{IVCO}(X), \leq)$ is a complete lattice with the smallest element $\tau_{\mathbf{0}}$ and the largest element $\tau_{\mathbf{1}}$.

Proposition 3.15. Let $(X, \tau)$ be an IVFTS, where $\tau \in \operatorname{IVGO}(X)$ and let $[\lambda, \mu] \in D(I)$. Then

$$
\tau_{[\lambda, \mu]}=\left\{A \in I^{X}: \tau(A) \geq[\lambda, \mu], \text { i.e., } \tau^{L}(A) \geq \lambda \text { and } \tau^{U}(A) \geq \mu\right\}
$$


is a Chang's fuzzy topology on $X$. In this case, $\tau_{[\lambda, \mu]}$ [resp. $\left.\tau_{\boldsymbol{\lambda}}\right]$ is called the $[\lambda, \mu]$-level [resp. $\lambda$-level] Chang's fuzzy topology on $X$ w.r.t. $\tau$.

Proof. Since $\tau \in \operatorname{IVGO}(X), \tau(\emptyset)=\tau(X)=\mathbf{1}$. Then

and

$$
\tau^{L}(\emptyset)=1 \geq \lambda, \tau^{U}(\emptyset)=1 \geq \mu
$$

$$
\tau^{L}(X)=1 \geq \lambda, \tau^{U}(X)=1 \geq \mu .
$$

Thus $\varnothing, X \in \tau_{[\lambda, \mu]}$. Let $A, B \in \tau_{[\lambda, \mu]}$. Then

$$
\tau^{L}(A) \geq \lambda, \tau^{U}(A) \geq \mu
$$

and

$$
\tau^{L}(B) \geq \lambda, \tau^{U}(B) \geq \mu .
$$

Since $\tau \in \operatorname{IVGO}(X)$

$$
\tau^{L}(A \cap B) \geq \tau^{L}(A) \wedge \tau^{L}(B) \geq \lambda
$$

and

$$
\tau^{U}(A \cap B) \geq \tau^{U}(A) \wedge \tau^{U}(B) \geq \mu .
$$

Thus $A \cap B \in \tau_{[\lambda, \mu]}$. Now let $\left\{A_{\alpha}\right\}_{\alpha \in \Gamma} \subset \tau_{[\lambda, \mu]}$. Then

$$
\tau^{L}\left(A_{\alpha}\right) \geq \lambda \text { and } \tau^{U}\left(A_{\alpha}\right) \geq \mu, \forall \alpha \in \Gamma .
$$

Since $\tau \in \operatorname{IVGO}(X)$,

$$
\tau^{L}\left(\bigcup_{\alpha \in \Gamma} A_{\alpha}\right) \geq \bigwedge_{\alpha \in \Gamma} \tau^{L}\left(A_{\alpha}\right) \geq \lambda
$$

and

$$
\tau^{U}\left(\bigcup_{\alpha \in \Gamma} A_{\alpha}\right) \geq \bigwedge_{\alpha \in \Gamma} \tau^{U}\left(A_{\alpha}\right) \geq \mu .
$$

Thus $\bigcup_{\alpha \in \Gamma} A_{\alpha} \in \tau_{[\lambda, \mu]}$. So $\tau_{[\lambda, \mu]}$ is a Chang's fuzzy topology on $X$. By the process of the proof of $\tau_{[\lambda, \mu]}$, it is clear that $\tau_{\boldsymbol{\lambda}}$ is a Chang's fuzzy topology on $X$.

Proposition 3.16. Let $(X, \tau)$ be an IVFTS and let $\left\{\tau_{[\lambda, \mu]}\right\}_{[\lambda, \mu] \in D(I)}$ be the family of all $[\lambda, \mu]$-level Chang's fuzzy topologies w.r.t. $\tau$. Then $\left\{\tau_{[\lambda, \mu]}\right\}_{[\lambda, \mu] \in D(I)}$ is dscending and for each $[\lambda, \mu] \in D\left(I_{o}\right), \tau_{[\lambda, \mu]}=\bigcap_{[a, b]<[\lambda, \mu]} \tau_{[a, b]}$.

In this case, $\left\{\tau_{[\lambda, \mu]}\right\}_{[\lambda, \mu] \in D\left(I_{o}\right)}$ is called the family of Chang's fuzzy topologies associated with the gradation of $\tau$.

Proof. Suppose $[a, b] \leq[\lambda, \mu]$. Then clearly $\tau_{[\lambda, \mu]} \subset \tau_{[a, b]}$. Thus $\left\{\tau_{[\lambda, \mu]}\right\}_{[\lambda, \mu] \in D(I)}$ is a descending family of Chang's fuzzy topologies. So

$$
\tau_{[\lambda, \mu]} \subset \bigcap_{[a, b]<[\lambda, \mu]} \tau_{[a, b]}, \text { for each }[\lambda, \mu] \in D\left(I_{o}\right) .
$$


Assume that $A \notin \tau_{[\lambda, \mu]}$. Then $\tau^{L}(A)<\lambda$ or $\tau^{U}(A)<\mu$. Thus $\exists[a, b] \in$ $D\left(I_{o}\right)$ such that $\tau^{L}(A)<a<\lambda$ or $\tau^{U}(A)<b<\mu$. So $A \notin \bigcap_{[a, b]<[\lambda, \mu]} \tau_{[a, b]}$. Hence $\bigcap_{[a, b]<[\lambda, \mu]} \tau_{[a, b]} \subset \tau_{[\lambda, \mu]}$. Therefore $\tau_{[\lambda, \mu]}=\bigcap_{[a, b]<[\lambda, \mu]} \tau_{[a, b]}$.

The following is the immediate result of Proposition 3.16.

Corollary 3.16. Let $(X, \tau)$ be an IVFTS and let $\left\{\tau_{\mathbf{r}}\right\}_{\mathbf{r} \in D(I)}$ be the family of all $\mathbf{r}$-level Chang's fuzzy topologies w.r.t. $\tau$. Then $\left\{\tau_{\mathbf{r}}\right\}_{\mathbf{r} \in D(I)}$ is descending and for each $\mathbf{r} \in D\left(I_{o}\right), \tau_{\mathbf{r}}=\bigcap_{\mathbf{s}<\mathbf{r}} \tau_{\mathbf{s}}$.

Proposition 3.17. Let $\left\{T_{[\lambda, \mu]}\right\}_{[\lambda, \mu] \in D\left(I_{o}\right)}$ be a nonempty descending family of Chang's fuzzy topologies on $X$. We define the mapping $\tau=$ $\left[\tau^{L}, \tau^{U}\right]: I^{X} \rightarrow D(I)$ as follows:

$$
\tau(A)=\bigvee\left\{[\lambda, \mu] \in D\left(I_{o}\right): A \in T_{[\lambda, \mu]}\right\}, \forall A \in I^{X}
$$

Then $\tau \in \operatorname{IVGO}(X)$. If, for each $[a, b] \in D\left(I_{o}\right)$,

$$
T_{[\lambda, \mu]}=\bigcap_{[a, b]<[\lambda, \mu]} T_{[a, b]},(3.2)
$$

then $\tau_{[\lambda, \mu]}=T_{[\lambda, \mu]}$ for each $[\lambda, \mu] \in D\left(I_{o}\right)$.

Proof. Since $T_{[\lambda, \mu]}$ is a Chang's fuzzy topology on $X, \emptyset, X \in T_{[\lambda, \mu]}$. Then, by the definition of $\tau$,

$$
\tau(\emptyset)=\tau(X)=\mathbf{1}
$$

Furthermore, $\tau^{L}(A) \leq \tau^{U}(A)$, for each $A \in I^{X}$. Thus $\tau$ satisfies the conditions (IVGO1) and (IVGO2).

For any $A_{i} \in I^{X}$, let $\tau\left(A_{i}\right)=\left[a_{i}, b_{i}\right]$ for $i=1,2$. Suppose $\tau\left(A_{i}\right)=\mathbf{0}$ for some $i$. Then clearly

$$
\tau\left(A_{1} \cap A_{2}\right) \geq \tau\left(A_{1}\right) \wedge \tau\left(A_{2}\right) .
$$

Thus, without loss of generality, suppose $\left[a_{i}, b_{i}\right]>\mathbf{0}$ for $i=1,2$. Let $[s, t] \leq \tau\left(A_{i}\right)$ for $i=1,2$ and let $\varepsilon>0$. Then, by the definition of $\tau$,

$\exists\left[\lambda_{1}, \mu_{1}\right],\left[\lambda_{2}, \mu_{2}\right] \in D\left(I_{o}\right)$ such that

$a_{i}-\varepsilon<\lambda_{i} \leq a_{i}, b_{i}-\varepsilon<\mu_{i} \leq b_{i}$ and $A_{i} \in T_{\left[\lambda_{i}, \mu_{i}\right]}$ for $i=1,2$.

Let $[\lambda, \mu]=\left[\lambda_{1}, \mu_{1}\right] \wedge\left[\lambda_{2}, \mu_{2}\right]$ and let $[a, b]=\left[a_{1}, b_{1}\right] \wedge\left[a_{2}, b_{2}\right]$. Then clearly $A_{1}, A_{2} \in T_{[\lambda, \mu]}$. Thus $A_{1} \cap A_{2} \in T_{[\lambda, \mu]}$. So

$$
\tau^{L}\left(A_{1} \cap A_{2}\right) \geq \lambda>a-\varepsilon>s-\varepsilon
$$

and

$$
\tau^{U}\left(A_{1} \cap A_{2}\right) \geq \mu>b-\varepsilon>t-\varepsilon .
$$


Since $\varepsilon>0$ is arbitrary,

$$
\tau^{L}\left(A_{1} \cap A_{2}\right) \geq s \text { and } \tau^{U}\left(A_{1} \cap A_{2}\right) \geq t .
$$

Hence $\tau\left(A_{1}, A_{2}\right) \geq[s, t]$, i.e., $\tau\left(A_{1} \cap A_{2}\right) \geq \tau\left(A_{1}\right) \wedge \tau\left(A_{2}\right)$. Therefore $\tau$ satisfies the condition (IVGO3).

Now suppose $\tau\left(A_{\alpha}\right)=\left[l_{\alpha}, m_{\alpha}\right]$ for each $\alpha \in \Gamma$ and let $[l, m]=$ $\bigwedge\left[l_{\alpha}, m_{\alpha}\right]$. Suppose $[l, m]=\mathbf{0}$. Then it is obvious that $\alpha \in \Gamma$

$$
\tau\left(\bigcup_{\alpha \in \Gamma} A_{\alpha}\right) \geq \bigwedge_{\alpha \in \Gamma} \tau\left(A_{\alpha}\right)
$$

Suppose $[l, m]>0$ and let $[l, m]>\varepsilon>\mathbf{0}$. Then $0<l-\varepsilon<l_{\alpha}$ and $0<m-\varepsilon<m_{\alpha}$ for each $\alpha \in \Gamma$. Thus $A_{\alpha} \in T_{[l-\varepsilon, m-\varepsilon]}, \forall \alpha \in \Gamma$. Since $T_{[l-\varepsilon, m-\varepsilon]}$ is a Chang's fuzzy topology, $\bigcup_{\alpha \in \Gamma} A_{\alpha} \in T_{[l-\varepsilon, m-\varepsilon]}$. So

$$
\tau\left(\bigcup_{\alpha \in \Gamma} A_{\alpha}\right) \geq[l-\varepsilon, m-\varepsilon] .
$$

Since $\varepsilon>0$ is arbitrary,

$$
\tau\left(\bigcup_{\alpha \in \Gamma} A_{\alpha}\right) \geq[l, m]=\bigwedge_{\alpha \in \Gamma} \tau\left(A_{\alpha}\right) .
$$

Hence $\tau$ satisfies the condition (IVGO.4). Therefore $\tau \in \operatorname{IVGO}(X)$.

Finally, suppose $\left\{T_{[\lambda, \mu]}\right\}_{[\lambda, \mu] \in D\left(I_{o}\right)}$ satisfies the condition (3.2) and let $A \in T_{[\lambda, \nu]}$. Then clearly $\tau(A) \geq[\lambda, \mu]$. Thus $A \in \tau_{[\lambda, \mu]}$. So $T_{[\lambda, \mu]} \subset \tau_{[\lambda, \mu]}$. Now let $A \in \tau_{[\lambda, \mu]}$. Then $\tau(A) \geq[\lambda, \mu]$. Thus, by the definition of $\tau$,

$$
\bigvee\left\{[a, b] \in D\left(I_{o}\right): A \in T_{[a, b]}\right\}=[s, t] \geq[\lambda, \mu] .
$$

Let $\varepsilon>0$. Then $\exists[a, b] \in D\left(I_{o}\right)$ such that

$$
s-\varepsilon<a, t-\varepsilon<b \text { and } A \in T_{[a, b]} .
$$

Thus

$$
\lambda-\varepsilon \leq s-\varepsilon<a, \mu-\varepsilon \leq t-\varepsilon<b \text { and } A \in T_{[a, b]} .
$$

So $A \in T_{[\lambda-\varepsilon, \mu-\varepsilon]}$. Since $\varepsilon>0$ is arbitrary, by the condition (3.2), $A \in T_{[\lambda, \mu]}$. Hence $\tau_{[\lambda, \mu]} \subset T_{[\lambda, \mu]}$. Therefore $\tau_{[\lambda, \mu]}=T_{[\lambda, \mu]}$. This completes the proof.

The followings are the immediate results of Corollary 3.16 and Proposition 3.17 .

Corollary 3.17-1. Let $\tau, \eta \in \operatorname{IVGO}(X)$. Then $\tau=\eta$ if and only if $\tau_{[\lambda, \mu]}=\eta_{[\lambda, \mu]}, \forall[\lambda, \mu] \in D\left(I_{o}\right)$. 
Corollary 3.17-2. Let $\left\{T_{\mathbf{r}}\right\}_{\mathbf{r} \in D\left(I_{o}\right)}$ be a nonempty dscending family of Chang's fuzzy topologices on $X$ and let $\tau: I^{X} \rightarrow D(I)$ be a mapping defined as follows: For each $A \in I^{X}$,

$$
\tau(A)=\bigvee\left\{\mathbf{r} \in D\left(I_{o}\right): A \in T_{\mathbf{r}}\right\}
$$

Then $\tau \in \operatorname{IVGO}(X)$. If, for each $\mathbf{r} \in D\left(I_{o}\right)$,

$$
T_{\mathbf{r}}=\bigcap_{\mathbf{s}<\mathbf{r}} T_{\mathbf{s}}
$$

then $\tau_{\mathbf{r}}=T_{\mathbf{r}}$ for all $\mathbf{r} \in D\left(I_{o}\right)$.

Proposition 3.18. Let $(X, T)$ be a Chang's fuzzy topological space. For each $[\lambda, \mu] \in D\left(I_{o}\right)$, we define a mapping $T^{[\lambda, \mu]}: I^{X} \rightarrow D(I)$ as follows: For each $A \in I^{X}$,

$$
T^{[\lambda, \mu]}(A)=\left\{\begin{array}{l}
\mathbf{1} \text { if } A=\emptyset \text { or } A=X, \\
{[\lambda, \mu] \text { if } A \in T \backslash\{\emptyset, X\},} \\
\mathbf{0} \text { otherwise. }
\end{array}\right.
$$

Then $T^{[\lambda, \mu]} \in \operatorname{IVGO}(X)$ such that $\left(T^{[\lambda, \mu]}\right)_{[\lambda, \mu]}=T$.

In this case, $T^{[\lambda, \mu]}$ [resp. $\left.T^{\boldsymbol{\lambda}}\right]$ is called a $[\lambda, \mu]$-th [resp. $\boldsymbol{\lambda}$-th] intervalvalued gradation [in short, $I V G]$ an $X$, and $\left(X, T^{[\lambda, \mu]}\right)\left[\operatorname{resp} .\left(X, T^{\boldsymbol{\lambda}}\right)\right]$ is called a $[\lambda, \mu]$-th [resp. $\lambda$-th] interval-valued graded fuzzy topological space.

Proof. By the definition of $T^{[\lambda, \mu]}, T^{[\lambda, \mu]^{L}}(A) \leq T^{[\lambda, \mu]^{U}}(A), \forall A \in I^{X}$. Then (IVGO1) holds. Also, it is clear that (IVGO2) holds.

Let $A_{i} \in I^{X}, i=1,2$. Suppose $A_{i}=\varnothing$ for some $i$. Then $A_{1} \cap A_{2}=\emptyset$. Thus

$$
T^{[\lambda, \mu]}\left(A_{1} \cap A_{2}\right)=\mathbf{1} \geq T^{[\lambda, \mu]}\left(A_{1}\right) \wedge T^{[\lambda, \mu]}\left(A_{2}\right) .
$$

Suppose $A_{i}=X$, for some $i$ (say $A_{1}$ ). Then $A_{1} \cap A_{2}=A_{2}$. Thus

$$
T^{[x, \mu]}\left(A_{1} \cap A_{2}\right)=T^{[\lambda, \mu]}\left(A_{2}\right) \geq T^{[\lambda, \mu]}\left(A_{1}\right) \wedge T^{\lambda, \mu}\left(A_{2}\right) .
$$

Suppose $A_{1}, A_{2} \in T \backslash\{\emptyset, X\}$. Then $A_{1} \cap A_{2} \in T$. Thus

$$
T^{[x, \mu]}\left(A_{1} \cap A_{2}\right) \geq[\lambda, \mu]=T^{[\lambda, \mu]}\left(A_{1}\right) \wedge T^{[\lambda, \mu]}\left(A_{2}\right) .
$$

Suppose $A_{i} \in I^{X}-T$ for some $i$ (say $A_{1}$ ) Then $T^{[\lambda, \mu]}\left(A_{1}\right)=\mathbf{0}$. Thus

$$
T^{[\lambda, \mu]}\left(A_{1} \cap A_{2}\right) \geq \mathbf{0}=T^{[\lambda, \mu]}\left(A_{1}\right) \wedge T^{[\lambda, \mu]}\left(A_{2}\right) .
$$

In all cases, $T^{[\lambda, \mu]}$ satisfies the condition (IVGO3).

Let $\left\{A_{\alpha}\right\}_{\alpha \in P} \subset I^{X}$. Suppose $A_{\alpha_{0}}=\emptyset$ for some $\alpha_{0} \in \Gamma$. Then

$$
\bigcup_{\alpha \in \Gamma} A_{\alpha}=\bigcup_{\alpha \in \Gamma\left(\alpha \neq \alpha_{0}\right)} A_{\alpha}
$$


Thus

$$
\bigwedge_{\alpha \in \Gamma} T^{[\lambda, \mu]}\left(A_{\alpha}\right)=\bigwedge_{\alpha \in \Gamma\left(\alpha \neq \alpha_{0}\right)} T^{[\lambda, \mu]}\left(A_{\alpha}\right) .\left[\text { Since } T^{\lambda, \mu}\left(A_{\alpha_{0}}\right)=\mathbf{1}\right]
$$

So, without loss of generality, assume that $A_{\alpha} \neq \emptyset \forall \alpha \in \Gamma$.

Suppose $A_{\alpha_{0}}=X$ for some $\alpha_{0}=\Gamma$. Then

$$
T^{[\lambda, \mu]}\left(\bigcup_{\alpha \in \Gamma} A_{\alpha}\right)=T^{[\lambda, \mu]}(X)=\mathbf{1} \geq \bigwedge_{\alpha \in \Gamma} T^{[\lambda, \mu]}\left(A_{\alpha}\right) .
$$

Suppose $A_{\alpha} \in T \backslash\{\emptyset, X\} \forall \alpha \in \Gamma$. Then clearly $\left(\bigcup_{\alpha \in \Gamma} A_{\alpha} \in T\right.$. Thus

$$
T^{[\lambda, \mu]}\left(\bigcup_{\alpha \in \Gamma} A_{\alpha}\right) \geq[\lambda, \mu]=\left(\bigwedge_{\alpha \in \Gamma} T^{[\lambda, \mu]}\left(A_{\alpha}\right) .\right.
$$

Suppose $A_{\alpha_{0}} \in I^{X}-T$ for some $\alpha_{0} \in \Gamma$. Then

$$
T^{[\lambda, \mu]}\left(\bigcup_{\alpha \in \Gamma} A_{\alpha}\right) \geq \mathbf{0}=T^{[\lambda, \mu]}\left(A_{\alpha_{0}}\right)=\bigwedge_{\alpha \in \Gamma} T^{[\lambda, \mu]}\left(A_{\alpha}\right) .
$$

In all cases, $T^{[\lambda, \mu]}$ satisfies the condition (IVGO4). Hence $T^{[\lambda, \mu]} \in$ $\operatorname{IVGO}(X)$.

By the above result and Proposition 3.15,

$$
\left(T^{[\lambda, \mu]}\right)_{[\lambda, \mu]}=\left\{A \in I^{X}: T^{[\lambda, \mu]}(A) \geq[\lambda, \mu]\right\}=T .
$$

From the process of the above proof, it can be easily seen that the remainder holds.

\section{Interval-valued gradation of clopenness}

Definition 4.1. A mapping $\tau: I^{X} \rightarrow D(I)$ is called an interval-valued gradation of clopenness (in short, IVGCO) on $X$ if $\tau \in \operatorname{IVGO}(X) \cap \operatorname{IVGC}(X)$. We will denote the set of all IVGCOs on $X$ as $\operatorname{IVGCO}(X)$. It is clear that $\tau_{\mathbf{0}}, \tau_{\mathbf{1}} \in \operatorname{IVGCO}(\mathrm{X})$.

Example 4.1. Let $[\lambda, \mu] \in D(I)$ be fixed. We define the mapping $\tau: I^{X} \rightarrow D(I)$ as follows : For each $A \in I^{X}$,

$$
\tau(A)= \begin{cases}1 & \text { if } A=\emptyset \text { or } A=X \\ {[\lambda, \mu]} & \text { if } A \neq \emptyset \text { and } A \neq X\end{cases}
$$

Then it is obvious that $\tau \in \operatorname{IVGCO}(\mathrm{X})$. In this case, $\tau$ is called an interval-valued constant gradation and we will denote it by $[\lambda, \mu]$.

The following is the characterization of IVGCO.

Theorem 4.2. $\tau \in \operatorname{IVGCO}(X)$ if and only if 
(i) $\tau^{L}(A) \leq \tau^{U}(A), \forall A \in I^{X}$,

(ii) $\tau(\emptyset)=\tau(X)=\mathbf{1}$

(iii) $\tau\left(\bigcap_{\alpha \in \Gamma} A_{\alpha}\right) \geq \bigwedge_{\alpha \in \Gamma} \tau\left(A_{\alpha}\right), \forall\left\{A_{\alpha}\right\}_{\alpha \in \Gamma} \subset I^{X}$,

(iv) $\tau\left(\bigcup_{\alpha \in \Gamma} A_{\alpha}\right) \geq \bigwedge_{\alpha \in \Gamma} \tau\left(A_{\alpha}\right), \forall\left\{A_{\alpha}\right\}_{\alpha \in \Gamma} \subset I^{X}$.

Proof. From Definitions 3.3, 3.8 and 4.1, it is obvious.

Definition 4.3. In Proposition 3.9, for each $\tau \in \operatorname{IVGO}(\mathrm{X}), \mathcal{F}_{\tau}$ is called an inter-val-valued conjugate gradation of $\tau$. By Proposition 3.9(c), $\tau$ is the interval-valued conjugate gradation of $\mathcal{F}_{\tau}$.

It is clear that if $\tau \in \operatorname{IVGCO}(\mathrm{X}), \mathcal{F}_{\tau}=\tau$.

The following gives a nice IVGCO.

Proposition 4.4. We define the mapping $\sigma: I^{X} \rightarrow D(I)$ as follows:

$$
\sigma(A)=\left\{\begin{array}{l}
\mathbf{1} \\
{\left[\bigwedge_{x \in \operatorname{supp}(\mathrm{A})} A(x), \bigwedge_{x \in \operatorname{supp}(\mathrm{A})}^{\text {if } A=\emptyset,} A(x)\right] \text { if } A \neq \emptyset,}
\end{array}\right.
$$

for each $A \in I^{X}$, where $\operatorname{supp}(\mathrm{A})=\{x \in X: A(x)>0\}$. Then $\sigma \in \operatorname{IVGCO}(X)$. In this case, $\sigma$ is called the interval-valued support gradation.

Proof. It is obvious that $\sigma(\emptyset)=\sigma(X)=1$ and $\sigma^{L}(A) \leq \sigma^{U}(A)$ for each $A \in I^{X}$.

Let $\left\{A_{\alpha}\right\}_{\alpha \in \Gamma} \subset I^{X}$, let $\lambda=\sigma\left(\bigcup_{\alpha \in \Gamma} A_{\alpha}\right)$ and let $\lambda_{\alpha}=\sigma\left(A_{\alpha}\right) \forall \alpha \in \Gamma$.

Suppose $\bigwedge_{\alpha \in \Gamma} \lambda_{\alpha}=\mu>\lambda$ and let $x \in \operatorname{supp}\left(\bigcup_{\alpha \in \Gamma} \mathrm{A}_{\alpha}\right)$. Since $\operatorname{supp}\left(\bigcup_{\alpha \in \Gamma} \mathrm{A}_{\alpha}\right)=$ $\bigcup_{\alpha \in \Gamma} \operatorname{supp}\left(\mathrm{A}_{\alpha}\right), \exists \alpha_{0} \in \Gamma$ such that $x \in \operatorname{supp}\left(A_{\alpha_{0}}\right)$. Thus

$A_{\alpha_{0}}(x) \geq \bigwedge\left\{A_{\alpha_{0}}(y): y \in \operatorname{supp}\left(\mathrm{A}_{\alpha_{0}}\right)\right\}=\lambda_{\alpha_{0}} \geq \mu$.

So $\left(\bigcup_{\alpha \in \Gamma} A_{\alpha}\right)(x) \geq \mu$ and hence $\sigma\left(\bigcup_{\alpha \in \Gamma} A_{\alpha}\right) \geq \mu$. This is a contradiction

from the fact that $\sigma\left(\bigcup_{\alpha \in \Gamma} A_{\alpha}\right)=\lambda<\mu$. Therefore $\sigma\left(\bigcup_{\alpha \in \Gamma} A_{\alpha}\right) \geq \bigwedge_{\alpha \in \Gamma} \sigma\left(A_{\alpha}\right)$ 
Now let $\lambda=\bigwedge\left\{\left(\bigcap_{\alpha \in \Gamma} A_{\alpha}\right)(x): x \in \operatorname{supp}\left(\bigcap_{\alpha \in \Gamma} \mathrm{A}_{\alpha}\right)\right\}$. Then

$$
\begin{aligned}
& \lambda=\bigwedge\left\{\bigwedge_{\alpha \in \Gamma} A_{\alpha}(x): x \in \operatorname{supp}\left(\bigcap_{\alpha \in \Gamma} \mathrm{A}_{\alpha}\right)\right\} \\
& =\bigwedge_{\alpha \in \Gamma}\left(\bigwedge\left\{A_{\alpha}(x): x \in \operatorname{supp}\left(\bigcap_{\alpha \in \Gamma} \mathrm{A}_{\alpha}\right)\right\}\right) \\
& \geq \bigwedge_{\alpha \in \Gamma}\left(\bigwedge\left\{A_{\alpha}(x): x \in \operatorname{supp}\left(\mathrm{A}_{\alpha}\right)\right\}\right) .
\end{aligned}
$$

Thus, by the definition of $\sigma$,

$$
\begin{aligned}
& \sigma\left(\bigcap_{\alpha \in \Gamma} A_{\alpha}\right)=\lambda \\
& \geq \bigwedge_{\alpha \in \Gamma}\left[\bigwedge\left\{A_{\alpha}(x): x \in \operatorname{supp}\left(\mathrm{A}_{\alpha}\right)\right\}, \bigwedge\left\{\mathrm{A}_{\alpha}(\mathrm{x}): \mathrm{x} \in \operatorname{supp}\left(\mathrm{A}_{\alpha}\right)\right\}\right] \\
& =\bigwedge_{\alpha \in \Gamma} \sigma\left(A_{\alpha}\right) .
\end{aligned}
$$

Hence $\sigma \in \operatorname{IVGCO}(\mathrm{X})$.

Remark 4.4. Let $\sigma$ be the IVGCO on $X$ given by Proposition 4.4. Then its conjugate gradation $\mathcal{F}_{\sigma}$ is given by, for each $A \in I^{X}$,

$$
\begin{aligned}
& \mathcal{F}_{a}(A)=\sigma\left(A^{c}\right) \\
& =\left[\bigwedge\left\{A^{c}(x): x \in \operatorname{supp}\left(A^{c}\right)\right\}, \bigwedge\left\{A^{c}(x): x \in \operatorname{supp}\left(A^{c}\right)\right\}\right] \\
& =[\bigwedge\{1-A(x): A(x) \neq 0\}, \bigwedge\{1-A(x) \neq 0\}] \\
& =[1-\bigvee\{A(x): A(x) \neq 0\}, 1-\bigvee\{A(x): A(x) \neq 0\}] .
\end{aligned}
$$

Example 4.4. Let $X$ be a set with two points at least. We define the mapping $\delta: I^{X} \rightarrow D(I)$ as follows : For each $A \in I^{X}$,

$$
\sigma(A)= \begin{cases}\mathbf{1} & \text { if } A=\emptyset \text { or } A=X \text { or } \operatorname{supp}(A)=X \\ 0 & \text { if } \operatorname{supp}(A) \neq X .\end{cases}
$$

Then it can be easily seen that $\delta \in \operatorname{IVGO}(\mathrm{X})$. For a fixed point $p \in X$ and for $\mathrm{n}=1,2, \cdots$, we define the mapping $G_{n}: X \rightarrow I$ as follows : For each $x \in X$,

$$
G_{n}(x)=\left\{\begin{array}{l}
\frac{1}{n} \text { if } x \neq p \\
1 \text { if } x=p
\end{array}\right.
$$

Then clearly $\left\{G_{n}\right\}_{n \in \mathbb{N}} \subset I^{X}$ and $\delta\left(G_{n}\right)=\mathbf{1} \forall n \in \mathbb{N}$, where $\mathbb{N}$ denotes the set of all positive integers. But, $\delta\left(\bigcap_{n \in \mathbb{N}} G_{n}\right)=\mathbf{0}$. Thus $\delta\left(\bigcap_{n \in \mathbb{N}} G_{n}\right)<$ 
$\mathbf{1}=\bigwedge_{n \in \mathbb{N}} \delta\left(G_{n}\right)$. So $\delta \notin \operatorname{IVGC}(\mathrm{X})$. Hence $\delta \notin \operatorname{IVGCO}(\mathrm{X})$.

The following gives a sufficient condition to be an IVGCO.

Proposition 4.5. Let $\delta: I^{X} \rightarrow D(I)$ be a mapping. Consider the following conditions :
(a) $\sigma^{L}(A) \leq \sigma^{U}(A), \forall A \in I^{X}$,
(b) $\sigma(\emptyset)=\mathbf{1}$,
(c) $\sigma(A)=\sigma\left(A^{c}\right), \forall A \in I^{X}$,
(d) $\sigma\left(\bigcup_{\alpha \in \Gamma} A_{\alpha}\right) \geq \bigwedge_{\alpha \in \Gamma} \sigma\left(A_{\alpha}\right), \forall\left\{A_{\alpha}\right\}_{\alpha \in \Gamma} \subset I^{X}$,
(e) $\sigma\left(\bigcap_{\alpha \in \Gamma} A_{\alpha}\right) \geq \bigwedge_{\alpha \in \Gamma} \sigma\left(A_{\alpha}\right), \forall\left\{A_{\alpha}\right\}_{\alpha \in \Gamma} \subset I^{X}$.

If $\sigma$ satisfies the condition (a) (d) or (a) $\sim(\mathrm{c})$ and (e), then $\sigma \in \operatorname{IVGCO}(\mathrm{X})$.

Proof. The condition (e) is deduced from the condition (b) and (c). Also the condition (d) is deduced from the condition (b) and (e). Hence, by Theorem $4.2, \sigma \in \operatorname{IVGCO}(\mathrm{X})$.

The following is the immediate result of Theorem 4.2 and Proposition 4.5 .

Corollary 4.5. If $\sigma \in \operatorname{IVGO}(\mathrm{X})$ or $\sigma \in \operatorname{IVGC}(\mathrm{X})$, and $\sigma(A)=\sigma\left(A^{c}\right)$ for each $A \in I^{X}$, then $\sigma \in \operatorname{IVGCO}(\mathrm{X})$.

The following is the immediate result of Definition 4.3 and Proposition 3.11 .

Proposition 4.6. Let $\left\{\tau_{\alpha}\right\}_{\alpha \in \Gamma} \subset \operatorname{IVGC}(\mathrm{X})$ [resp. IVGCO(X)]. Then $\bigcap_{\alpha \in \Gamma} \tau_{\alpha} \in \operatorname{IVGC}(\mathrm{X})[$ resp. $\operatorname{IVGCO}(\mathrm{X})]$.

Definition 4.7. Let $\left\{\tau_{\alpha}\right\}_{\alpha \in \Gamma} \subset \operatorname{IVGO}(\mathrm{X})$. Then the union of $\left\{\tau_{\alpha}\right\}_{\alpha \in \gamma}$, denoted by $\bigcup_{\alpha \in \Gamma} \tau_{\alpha}$, is defined as follows : For each $A \in I^{X}$,

$$
\left(\bigcup_{\alpha \in \Gamma} \tau_{\alpha}\right)(A)=\left[\bigvee_{\alpha \in \Gamma} \tau_{\alpha}{ }^{L}(A), \bigvee_{\alpha \in \Gamma} \tau_{\alpha}^{U}(A)\right]
$$

The following example shows that the union of two IVGCOs is not, in general, an IVGO(IVGC) even they are conjugate. 
Example 4.7. Let $X$ be a set with two points at least. Let $\{M, N\}$ be a partition of $X$, let $\frac{1}{2}<\lambda<1$ and let $\mu=1-\lambda$. Consider two fuzzy sets $\mathrm{A}$ and $\mathrm{B}$ in $X$ defined as follows : For each $x \in X$,

$$
A(x)= \begin{cases}\mathbf{0} & \text { if } x \in M \\ \lambda & \text { if } x \in N\end{cases}
$$

and

$$
B(x)=\left\{\begin{array}{l}
\mu \text { if } x \in M \\
0 \text { if } x \in N .
\end{array}\right.
$$

Then $A \cup B$ is the fuzzy set in $X$ given by, for each $x \in X$,

$$
(A \cup B)(x)=\left\{\begin{array}{l}
\mu \text { if } x \in M \\
\lambda \text { if } x \in N .
\end{array}\right.
$$

Let $\sigma$ be the interval-valued support gradation and let $\delta$ be its conjugate gradation. Then

$$
(\sigma \cup \delta)(A \cup B)=[\mu, \mu],
$$

and

$$
(\sigma \cup \delta)(A)=[\lambda, \lambda],(\sigma \cup \delta)(B)=[1-\mu, 1-\mu]=[\lambda, \lambda] .
$$

Since $\frac{1}{2}<\lambda<1$ and $\mu=1-\lambda, \mu<\lambda$. Thus

$(\sigma \cup \delta)(A \cup B)=[\mu, \mu]<[\lambda, \lambda]=(\sigma \cup \delta)(A) \wedge(\sigma \cup \delta)(B)$ So $\sigma \cup \delta \notin \operatorname{IVGCO}(\mathrm{X})$.

Definition 4.8[9]. Let $(X, T)$ be a Chang's fuzzy topological space. Then the fuzzy space $X$ (the fuzzy topology $T$ ) is said to be interpreservative[resp. super 0-dimensional] if the intersection of each family of open sets is open [resp. each open set is closed or equivalently if the family of closed sets in $X$ agrees with $T$.

It is clear that if $X$ is super 0-dimensional, then $X$ is interpreservative.

Definition 4.9. Let $\sigma \in \operatorname{IVGO}(\mathrm{X})$ and let $\mathrm{T}$ be a Chang's fuzzy topology on $X$. We define the mapping $\sigma^{*}: I^{X} \rightarrow B(I)$ as follows : For each $A \in I^{X}$,

$$
\sigma^{*}(A)=\left\{\begin{array}{l}
\sigma(A) \text { if } A \in T \\
\mathbf{0} \text { otherwise }
\end{array}\right.
$$

Then $\sigma^{*}$ is called the deduced gradation from $\sigma$ and $T$. 
It is clear that $\sigma^{*} \in \operatorname{IVGO}(\mathrm{X})$ and its $[\lambda, \mu]$-level $\sigma_{[\lambda, \mu]}^{*}$ is $\sigma_{[\lambda, \mu]}^{*}=$ $\sigma_{[\lambda, \mu]}=\sigma_{[\lambda, \mu]} \cap T$ for each $[\lambda, \mu] \in D(I)$.

The following is the immediate result of Definitions 4.8 and 4.9 .

Proposition 4.10. Let $\sigma \in \operatorname{IVGO}(\mathrm{X})$ and let $\mathrm{T}$ be a Chang's fuzzy topology. Then

(a) If $\sigma^{*}$ is deduced gradation from $\sigma$ and $T$, then $\sigma^{*} \in \operatorname{IVGCO}(\mathrm{X})$.

(b) If $\delta$ is the conjugate gradation of $\sigma$ and $T$ is super 0-dimensional, then $\delta^{*}$ is the conjugate gradation of $\sigma^{*}$ and hence $\delta^{*} \in \operatorname{IVGCO}(\mathrm{X})$.

Example 4.10. Let $\sigma$ be the interval-valued support gradation on $\mathbb{R}$, let $\delta$ be its conjugate and let $T$ be the laminated indiscrete topology on $\mathbb{R}[13]$, i.e., $T$ is constituted by the constant mappings on $\mathbb{R}$. Then clearly $\sigma$ and $T$ satisfies (b) of Proposiotion 4.10. Let $f_{\alpha} \in T$ be the constant mapping given by $f_{\alpha}(x)=\alpha$ for each $x \in X$. Then, the deduced gradation gradation $\sigma^{*}$ from $\sigma$ and $T$ is given by : For each $A \in I^{X}$,

$$
\sigma(A)=\left\{\begin{array}{l}
\mathbf{1} \text { if } A=\emptyset \text { or } A=X, \\
{[\alpha, \alpha] \text { if } A=f_{\alpha} \in T \text { and } \alpha \neq 0,} \\
\mathbf{0} \text { otherwise. }
\end{array}\right.
$$

Then

$$
\begin{aligned}
& \delta^{*}(\emptyset)=\delta^{*}(X)=\mathbf{1}, \\
& \delta^{*}\left(f_{\alpha}\right)=\delta\left(f_{\alpha}\right)=\sigma\left(1-f_{\alpha}\right)=[1-\alpha, 1-\alpha] \\
& =\sigma^{*}\left(1-f_{\alpha}\right)=\sigma^{*}\left(f_{\alpha}^{c}\right), \text { if } \alpha \neq 1 .
\end{aligned}
$$

By the definition of $T$, it is clear that $A \in T$ if and only if $A^{c}(=1-A) \in$ $T$. Thus, for $A \notin T, \delta^{*}(A)=\sigma^{*}\left(A^{c}\right)=\mathbf{0}$. So $\sigma^{*}$ and $\delta^{*}$ are conjugate.

Definition 4.11. Let $\tau, \eta \in \operatorname{IVGO}(X)$. Then we say that $\tau$ is equivalent to $\eta, \mathcal{F}$ denoted by $\tau \approx \eta$, if their families $[\lambda, \mu]$-levels agree, i.e., $\left\{\tau_{[\lambda, \mu]}\right\}_{[\lambda, \mu] \in D(I)}=\left\{\eta_{[a, b]}\right\}_{[a, b] \in D(I)}$.

Proposition 4.12. Let $\sigma \in \operatorname{IVGO}(\mathrm{X})$ [resp. $\operatorname{IVGC}(\mathrm{X})]$ and let $\varphi$ : $I \rightarrow I$ be an increasing continuous mapping with $\varphi(1)=1$. Then $\varphi \circ \sigma=\left[\varphi \circ \sigma^{L}, \varphi \circ \sigma^{U}\right] \in \operatorname{IVGO}(\mathrm{X})[$ resp. $\operatorname{IVGC}(\mathrm{X})]$. Moreover, if $\varphi$ is strictly increasing, then $\sigma \approx \varphi \circ \sigma$. 
Proof. Suppose $\sigma \in \operatorname{IVGO}(X)$. Then it is clear that the condition (IVGO1) holds. On the other hand,

$$
\begin{aligned}
& (\varphi \circ \sigma)(\emptyset)=\left[\left(\varphi \circ \sigma^{L}\right)(\emptyset),\left(\varphi \circ \sigma^{U}\right)(\emptyset)\right] \\
& =\left[\left(\varphi\left(\sigma^{L}\right)(\emptyset)\right),\left(\varphi\left(\sigma^{U}\right)(\emptyset)\right)\right] \\
& =[(\varphi(1),(\varphi(1)] \\
& =[1,1]=\mathbf{1} .
\end{aligned}
$$

Similarly, $(\varphi \circ \sigma)(X)=\mathbf{1}$. Thus the condition(IVGO2) holds.

Let $A, B \in I^{X}$. Then

$$
\begin{aligned}
& (\varphi \circ \sigma)(A \cap B)=\left[\left(\varphi \circ \sigma^{L}\right)(A \cap B),\left(\varphi \circ \sigma^{U}\right)(A \cap B)\right] \\
& \geq\left[\left(\varphi\left(\sigma^{L}(A \cap B)\right),\left(\varphi\left(\sigma^{U}(A \cap B)\right)\right]\right.\right. \\
& \geq\left[\left(\varphi\left(\sigma^{L}(A) \wedge \sigma^{L}(B)\right),\left(\varphi\left(\sigma^{U}(A) \wedge \sigma^{U}(B)\right)\right] .(4.1)\right.\right. \\
& {[\text { Since } \sigma \in \operatorname{IVGO}(X)]}
\end{aligned}
$$

Suppose $\sigma^{L}(A) \leq \sigma^{L}(B)$. Since $\varphi$ is increasing continuous,

$$
\varphi\left(\sigma^{L}(A)\right) \leq \varphi\left(\sigma^{L}(B)\right) .
$$

Thus

$$
\begin{aligned}
& \varphi\left(\sigma^{L}(A)\right) \leq \varphi\left(\sigma^{L}(B)\right)=\varphi\left(\sigma^{L}(A)\right)=\varphi\left(\sigma^{L}(A)\right) \wedge \varphi\left(\sigma^{L}(B)\right) \\
& =\left(\varphi \circ \sigma^{L}\right)(A) \wedge\left(\varphi \circ \sigma^{L}\right)(B) .(4.2)
\end{aligned}
$$

Similarly, we have

$$
\varphi\left(\sigma^{U}\right)(A) \wedge \sigma^{U}(B)=\left(\varphi \circ \sigma^{U}\right)(A) \wedge\left(\varphi \circ \sigma^{U}\right)(B) . \text { (4.3) }
$$

So, by $(4.1),(4,2)$ and $(4,3)$,

$(\varphi \circ \sigma)(A \cap B) \geq(\varphi \circ \sigma)(A) \wedge(\varphi \circ \sigma)(B)$.

Hence the condition (IVGO3) holds.

Now let $\left\{A_{\alpha}\right\}_{\alpha \in \Gamma} \subset I^{X}$ and let $[\lambda, \mu]=\bigwedge_{\alpha \in \Gamma} \sigma\left(A_{\alpha}\right)$.

Suppose $\exists \alpha_{0} \in \Gamma$ such that $[\lambda, \mu]=\sigma\left(A_{\alpha_{0}}\right)$. Then

$$
\begin{aligned}
& (\varphi \circ \sigma)\left(\bigcup_{\alpha \in \Gamma} A_{\alpha}\right)=\left[\varphi\left(\sigma^{L}\left(\bigcup_{\alpha \in \Gamma} A_{\alpha}\right)\right), \varphi\left(\sigma^{U}\left(\bigcup_{\alpha \in \Gamma} A_{\alpha}\right)\right)\right] \\
& \geq\left[\varphi\left(\bigwedge_{\alpha \in \Gamma} \sigma^{L}\left(A_{\alpha}\right)\right), \varphi\left(\bigwedge_{\alpha \in \Gamma} \sigma^{U}\left(A_{\alpha}\right)\right)\right][\text { Since } \sigma \in \operatorname{IVGO}(X)] \\
& =\left[\varphi\left(\sigma^{L}\left(A_{\alpha_{0}}\right)\right), \varphi\left(\sigma^{U}\left(A_{\alpha_{0}}\right)\right)\right][\text { By the hypothesis }] \\
& =\left[(\varphi \circ \sigma)\left(A_{\alpha_{0}}\right)\right] \\
& \geq \bigwedge_{\alpha \in \Gamma}(\varphi \circ \sigma)\left(A_{\alpha}\right) .
\end{aligned}
$$

Suppose $\nexists \alpha_{0} \in \Gamma$ such that $[\lambda, \mu]=\sigma\left(A_{\alpha_{0}}\right)$. Then $\lambda \in a c\left\{a^{L}\left(A_{\alpha}\right)\right.$ : $\alpha \in \Gamma\}$ and $\mu \in a c\left\{\sigma^{U}\left(A_{\alpha}\right): \alpha \in \Gamma\right\}$. Thus $\exists$ strictly decreasing sequences $\left\{\sigma^{L}\left(A_{n}\right)\right\}_{n=1}^{\infty}$ and $\left\{\sigma^{U}\left(A_{n}\right)\right\}_{n=1}^{\infty}$ such that they converge to $\lambda$ and $\mu$, respectively. So $\left\{\left(\varphi \circ \sigma^{L}\right)\left(A_{n}\right)\right\}_{n=1}^{\infty}$ and $\left\{\left(\varphi \circ \sigma^{U}\right)\left(A_{n}\right)\right\}_{n=1}^{\infty}$ 
are lower bounded sequences and thus they converge to their infimums, respectively. Hence

$$
\begin{aligned}
& \lim _{n \rightarrow \infty}\left(\varphi \circ \sigma^{L}\right)\left(A_{n}\right)=\bigwedge_{n}\left(\varphi \circ \sigma^{L}\right)\left(A_{n}\right) \\
& \geq \bigwedge_{\alpha \in \Gamma}\left(\varphi \circ \sigma^{L}\right)\left(A_{\alpha}\right)(4.4)
\end{aligned}
$$

Similarly, we have

$$
\lim _{n \rightarrow \infty}\left(\varphi \circ \sigma^{U}\right)\left(A_{n}\right) \geq \bigwedge_{\alpha \in \Gamma}\left(\varphi \circ \sigma^{L}\right)\left(A_{\alpha}\right) \text {. }
$$

On the other hand,

$$
\begin{aligned}
& (\varphi \circ \sigma)\left(\bigcup_{\alpha \in \Gamma} A_{\alpha}\right) \geq\left[\varphi\left(\bigwedge_{\alpha \in \Gamma} \sigma^{L}\left(A_{\alpha}\right)\right), \varphi\left(\bigwedge_{\alpha \in \Gamma} \sigma^{U}\left(A_{\alpha}\right)\right)\right][\text { Since } \sigma \in \operatorname{IVGO}(X)] \\
& =[\varphi(\lambda), \varphi(\mu)]\left[\text { Since }[\lambda, \mu]=\bigwedge_{\alpha \in \Gamma} \sigma\left(A_{\alpha}\right)\right] \\
& =\left[\varphi\left(\lim _{n \rightarrow \infty} \sigma^{L}\left(A_{n}\right)\right), \varphi\left(\lim _{n \rightarrow \infty} \sigma^{U}\left(A_{n}\right)\right)\right] \\
& =\left[\lim _{n \rightarrow \infty}\left(\varphi \circ \sigma^{L}\right)\left(A_{n}\right), \lim _{n \rightarrow \infty}\left(\varphi \circ \sigma^{U}\left(A_{n}\right)\right)\right] .(4.6) \\
& {[\text { Since } \varphi \text { is continuous }]}
\end{aligned}
$$

From (4.4), (4.5) and (4.6),

$$
(\varphi \circ \sigma)\left(\bigcup_{\alpha \in \Gamma} A_{\alpha}\right) \geq \bigwedge_{\alpha \in \Gamma}(\varphi \cdot \alpha)\left(A_{\alpha}\right)
$$

So $\varphi \circ \sigma$ satisfies the condition (IVGO4). Hence $\varphi \circ \sigma \in \operatorname{IVGO}(X)$.

Suppose $\sigma \in \operatorname{IVGC}(\mathrm{X})$. By the similar way, we can prove that

$$
(\varphi \circ \sigma)\left(\bigcap_{\alpha \in \Gamma} A_{\alpha}\right) \geq\left(\bigwedge_{\alpha \in \Gamma}\right)(\varphi \circ \alpha)\left(A_{\alpha}\right)
$$

for each $\left\{A_{\alpha}\right\}_{\alpha \in \Gamma} \subset I^{X}$. Also we can easily see that the remainders hold. Hence $\varphi \circ \sigma \in \operatorname{IVGC}(X)$.

The following example shows that the continuity condition for the mapping $\varphi$ in Proposition 4.12 cannot be removed. The following is the modification of Example 2.16 in [9].

Example 4.12. Let $\delta^{*}$ be same as in Example 4.10. Let $\varphi: I \rightarrow I$ be the mapping defined as follows : For each $x \in I$,

$$
\varphi(x)=\left\{\begin{array}{l}
\frac{1}{2} x \text { if } x<\frac{1}{2}, \\
\frac{1}{2} \text { if } x=\frac{1}{2}, \\
\frac{1}{2} x+\frac{1}{2} \text { if } x>\frac{1}{2} .
\end{array}\right.
$$

Then $\varphi$ is strictly increasing and $\varphi(1)=1$. But it is not continuous at $x=\frac{1}{2}$. We will show that $\varphi \circ \delta^{*}$ is not an IVGO : 
Consider a strictly increasing sequence $\left\{k_{n}\right\}_{n=1}^{\infty}$ such that $\lim _{n \rightarrow \infty} k_{n}=$ $\frac{1}{2}$ and $0 \leq k_{n} \leq \frac{1}{2} \forall n \in \mathbb{N}$. For each $n \in \mathbb{N}$ we define the contant mapping $K_{n}: \mathbb{R} \rightarrow I$ as follows : For each $x \in \mathbb{R}, K_{n}(x)=k_{n}$. Then $\delta^{*}\left(K_{n}\right)=\left[1-k_{n}, 1-k_{n}\right]$ and $\left\{1-k_{n}\right\}_{n=1}^{\infty}$ is a strictly decreasing sequence contained in I such that $\left(1-k_{n}\right)=\frac{1}{2}$. Thus

$$
\begin{aligned}
& \left(\varphi \circ \delta^{*}\right)\left(K_{n}\right)=\left[\left(\varphi \circ \delta^{*^{L}}\right)\left(K_{n}\right),\left(\varphi \circ \delta^{*^{U}}\right)\left(K_{n}\right)\right] \\
& =\left[\varphi\left(1-k_{n}\right), \varphi\left(1-k_{n}\right)\right] \\
& =\left[\frac{1-k_{n}}{2}+\frac{1}{2}, \frac{1-k_{n}}{2}+\frac{1}{2}\right] .
\end{aligned}
$$

So $\varphi\left(1-k_{n}\right)_{n=1}^{\infty}$ is a strictly decreasing sequence such that $\lim _{n \rightarrow \infty} \varphi(1-$ $\left.k_{n}\right)=3 / 4$. Hence $\left(\varphi \circ \delta^{*}\right)\left(K_{n}\right) \geq \mathbf{3} / \mathbf{4}$, for $n=1,2, \cdots$.

On the other hand, $\bigcup_{n=1}^{\infty} K_{n}$ is the constant mapping $f_{\frac{1}{2}}: \mathbb{R} \rightarrow$ I given by $f_{\frac{1}{2}}(x)=\frac{1}{2}$ for each $x \in \mathbb{R}$. Then

$$
\left(\varphi \circ \delta^{*}\right)\left(\bigcup_{n=1}^{\infty} K_{n}\right)=[1-1 / 2,1-1 / 2]=\mathbf{1} / \mathbf{2} .
$$

Thus

$$
\left(\varphi \circ \delta^{*}\right)\left(\bigcup_{n=1}^{\infty} K_{n}\right)=[\varphi(1 / 2), \varphi(1 / 2)]=\mathbf{1} / \mathbf{2} .
$$

So

$$
\left(\varphi, \delta^{*}\right)\left(\bigcup_{n=1}^{\infty} K_{n}\right)<\bigwedge_{n=1}^{\infty}\left(\varphi \circ \delta^{*}\right) K_{n} .
$$

Hence $\varphi \circ \delta^{*} \notin \operatorname{IVGO}(\mathrm{X})$.

\section{Interval-valued fuzzy subspace.}

Definition 5.1[10]. Let $Y$ be a subset of $X$ and let $A \in I^{X}$. Then the restriction of $A$ on $Y$ is denoted by $\left.A\right|_{Y}$. For each $B \in I^{Y}$, the extension of $B$, on $X$, denoted by $B_{X}$, is defined by

$$
B_{X}(x)=\left\{\begin{array}{l}
B(x) \text { if } x \in Y, \\
0 \text { if } x \in X \backslash Y, \text { for each } x \in X
\end{array}\right.
$$

Proposition 5.2. Let $(X, \tau)$ be an IVFTS and let $Y \subset X$. We define the mapping $\tau_{Y}: I^{Y} \rightarrow D(I)$ as follows : For each $A \in I^{Y}$,

$$
\tau_{Y}(A)=\bigvee\left\{\tau(B): B \in I^{X} \text { and } \mathrm{A}=\left.\mathrm{B}\right|_{\mathrm{Y}}\right\} \text {. }
$$


Then $\tau_{Y} \in \operatorname{IVGO}(\mathrm{Y})$ and $\tau_{Y}(A) \geq \tau\left(A_{X}\right)$. In this case, the IVFTS $\left(Y, \tau_{Y}\right)$ is called a subspace of $(X, \tau)$ and $\tau_{Y}$ is called the induced IVGO on $Y$ from $(X, \tau)$.

Proof. For each $A \in I^{Y}$, let $B \in I^{X}$ such that $A=\left.B\right|_{Y}$. Then $\tau^{L}(B) \leq \tau^{U}(B)$

Thus

$$
\bigvee\left\{\tau^{L}(B): A=\left.B\right|_{Y}\right\} \leq \bigvee\left\{\tau^{U}(B): A=\left.B\right|_{Y}\right\}
$$

So, by the definition of $\tau_{Y}$,

$$
\tau_{Y}^{L}(A) \leq \tau_{Y}^{U}(A)
$$

Hence $\tau_{Y}$ satisfies the condition (IVGO1). It is obvious that (IVGO2) holds.

Let $A_{1}, A_{2} \in I^{Y}$. Then

$\tau_{Y}\left(A_{1} \cap A_{2}\right)=\bigvee\left\{\tau(B): B \in I^{X}\right.$ and $\left._{1} \cap \mathrm{A}_{2}=\left.\mathrm{B}\right|_{\mathrm{Y}}\right\}$.

Suppose $\tau_{Y}\left(A_{1}\right) \wedge \tau_{Y}\left(A_{2}\right)=\mathbf{0}$. Then clearly

$\tau_{Y}\left(A_{1} \cap A_{2}\right) \geq \mathbf{0}=\tau_{Y}\left(A_{1}\right) \wedge \tau_{Y}\left(A_{2}\right)$.

Suppose $\tau_{Y}\left(A_{1}\right) \wedge \tau_{Y}\left(A_{2}\right)>\mathbf{0}$. Let $\mathbf{0}<[\lambda, \mu]<\tau_{Y}\left(A_{1}\right) \wedge \tau_{Y}\left(A_{2}\right)$.

Then $\exists B_{i} \in I^{X}$ such that $A_{i}=\left.B_{i}\right|_{Y}$ and $\tau\left(B_{i}\right)>[\lambda, \mu], i=1,2$. Since $\tau \in \operatorname{IVGO}(X)$,

$$
\tau\left(B_{1} \cap B_{2}\right) \geq \tau\left(B_{1}\right) \wedge \tau\left(B_{2}\right)>[\lambda, \mu] .
$$

On the other hand,

$$
\left.\left(B_{1} \cap B_{2}\right)\right|_{Y}=\left(\left.B_{1}\right|_{Y}\right) \cap\left(\left.B_{2}\right|_{Y}\right)=A_{1} \cap A_{2} .
$$

Thus

$$
\tau_{Y}\left(A_{1} \cap A_{2}\right) \geq \tau\left(B_{1} \cap B_{2}\right)>[\lambda, \mu] .
$$

So, by the definition of $\tau_{Y}$,

$\tau_{Y}\left(A_{1} \cap A_{2}\right) \geq \tau_{Y}\left(A_{1}\right) \wedge \tau_{Y}\left(A_{2}\right)$.

In either cases,

$\tau_{Y}\left(A_{1} \cap A_{2}\right) \geq \tau_{Y}\left(A_{1}\right) \wedge \tau_{Y}\left(A_{2}\right)$.

Hence the condition (IVGO3) holds.

Now let $\left\{A_{\alpha}\right\}_{\alpha \in \Gamma} \subset I^{X}$. Then

$$
\tau_{Y}\left(\bigcup_{\alpha \in \Gamma} A_{\alpha}\right)=\bigvee\left\{\tau(B): B \in I^{X} \text { and } \bigcup_{\alpha \in \Gamma} \mathrm{A}_{\alpha}=\left.\mathrm{B}\right|_{\mathrm{Y}}\right\} .
$$

Suppose $\bigwedge_{\alpha \in \Gamma} \tau_{Y}\left(A_{\alpha}\right)=\mathbf{0}$. Then clearly

$$
\tau_{Y}\left(\bigcup_{\alpha \in \Gamma}^{\alpha \in \Gamma} A_{\alpha}\right) \geq \mathbf{0}=\bigwedge_{\alpha \in \Gamma} \tau_{Y}\left(A_{\alpha}\right)
$$

Suppose $\bigwedge_{\alpha \in \Gamma} \tau_{Y}\left(A_{\alpha}\right)>\mathbf{0}$ and let $\mathbf{0}<[\lambda, \mu]<\bigwedge_{\alpha \in \Gamma} \tau_{Y}\left(A_{\alpha}\right)$. Then $\tau_{Y}\left(A_{\alpha}\right)>[\lambda, \mu], \forall \alpha \in \Gamma$.

Thus $\exists B_{\alpha} \in I^{X}$ such that $A_{\alpha}=\left.B_{\alpha}\right|_{Y}$ and $\tau\left(B_{\alpha}\right)>[\lambda, \mu], \forall \alpha \in \Gamma$. 
So

$\tau\left(\bigcup_{\alpha \in \Gamma} B_{\alpha}\right) \geq[\lambda, \mu]$

On the other hand,

$$
\left.\left(\bigcup_{\alpha \in \Gamma} A_{\alpha}\right)\right|_{Y}=\bigcup_{\alpha \in \Gamma}\left(\left.A_{\alpha}\right|_{Y}\right)=\left(\bigcup_{\alpha \in \Gamma} B_{\alpha}\right) .
$$

Thus, by the definition of $\tau_{Y}$,

$$
\tau_{Y}\left(\bigcup_{\alpha \in \Gamma} A_{\alpha}\right) \geq \bigwedge_{\alpha \in \Gamma} \tau_{Y}\left(A_{2}\right)
$$

In either cases, $\tau_{Y}$ satisfies the condition (IVGO4). Hence $\tau \in \operatorname{IVGO}(Y)$.

It is clearly that $\tau_{Y}(A) \geq \tau\left(A_{X}\right), \forall A \in I^{Y}$.

Proposition 5.3. Let $\left(Y, \tau_{Y}\right)$ be an interval-valued fuzzy subspace of the IVFTS $(X, \tau)$ and let $A \in I^{Y}$. Then

(a) $\mathcal{F}_{\tau_{Y}}(A)=\bigvee\left\{\mathcal{F}_{\tau}(B): B \in I^{X}\right.$ and $\left.A=\left.B\right|_{Y}\right\}$.

(b) If $Z \subset Y \subset X$, then $\tau_{Z}=\left(\tau_{Y}\right)_{Z}$.

Proof. The proofs are very similar to that of Proposition 3.3 in (7). So they are omitted.

\section{Interval-valued gradation of preserving mappings}

Definition 6.1. Let $(X, \tau)$ and $(Y, \eta)$ be two IVSTSs and let $f: X \rightarrow Y$ be a mapping. Then $f$ is called an interval-valued gradation preserving mapping (in short, an IVGP-mapping) or interval-valued smooth continuous if for each $B \in I^{Y}$,

$$
\eta(B) \leq \tau\left(f^{-1}(B)\right) \text {, i.e., }\left[\eta^{L}(B), \eta^{U}(B)\right] \leq\left[\tau^{L}\left(f^{-1}(B)\right), \tau^{U}\left(f^{-1}(B)\right)\right] .
$$

Definition 6.1' $[7]$. Let $(X, \tau)$ and $(Y, \eta)$ be two STSs and let $f: X \rightarrow$ $Y$ be a mapping. Then $f$ is called a gradation preserving mapping (in short, an GP-mapping) or smooth continuous if for each $B \in I^{Y}, \eta(B) \leq$ $\tau\left(f^{-1}(B)\right.$.

Remark 6.1. (a) If a mapping $f:(X, \tau) \rightarrow(Y, \eta)$ is a GP-mapping, then $f:(X,[\tau, \tau]) \rightarrow(Y,[\eta, \eta])$ is an IVGP-mapping.

(b) If a mapping $f:(X, \tau) \rightarrow(Y, \eta)$ is an IVGP-mapping, then $f:\left(X, \tau^{L}\right) \rightarrow\left(Y, \eta^{L}\right)$ and $f:\left(X, \tau^{U}\right) \rightarrow\left(Y, \eta^{U}\right)$ are GP-mappings, respectively. 
Theorem 6.2. Let $(X, \tau)$ and $(Y, \eta)$ be two IVSTSs and let $f: X \rightarrow Y$ be a mapping. Then $f:(X, \tau) \rightarrow(Y, \eta)$ is an IVGP-mapping if and only if $f:\left(X, \tau_{[\lambda, \mu]}\right) \rightarrow\left(Y, \eta_{[\lambda, \mu]}\right)$ is continuous w.r.t. Chang, for each $[\lambda, \mu] \in D\left(I_{o}\right)$.

Proof. $(\Rightarrow)$ : Suppose $f$ is an IVGP-mapping. Let $[\lambda, \mu] \in D\left(I_{0}\right)$ and let $B \in \eta_{[\lambda, \mu]}$. Since $\eta \in \operatorname{IVGO}(Y), \eta(B) \geq[\lambda, \mu]$. Then, by the hypothesis, $\eta(B) \leq \tau\left(f^{-1}(B)\right)$. Thus

$$
\tau\left(f^{-1}(B)\right) \geq[\lambda, \mu]
$$

So $f^{-1}(B) \in \tau_{\lambda, \mu}$. Hence $f:\left(X, \tau_{[\lambda, \mu]}\right) \rightarrow\left(Y, \eta_{[\lambda, \mu]}\right)$ is continuous w.r.t. Chang.

$(\Leftarrow)$ : Suppose $f:\left(X, \tau_{[\lambda, \mu]}\right) \rightarrow\left(Y, \eta_{[\lambda, \mu]}\right)$ is continuous for each $[\lambda, \mu] \in D\left(I_{0}\right)$. Let $B \in I^{Y}$. If $\eta(B)=\mathbf{0}$, then clearly $\eta\left(B \leq \tau\left(f^{-1}(B)\right)\right)$. If $\eta(B)=[\lambda, \mu]$, then $B \in \eta_{[[\lambda, \mu]}$. Thus, by the hypothesis, $f^{-1}(B) \in$ $\tau_{[\lambda, \mu]}$. So $\tau\left(f^{-1}(B)\right) \geq[\lambda, \mu]=\eta(B)$. In either cases, $\eta(B) \leq \tau\left(f^{-1}(B)\right)$. Hence $f$ is an IVGP-mapping.

Theorem 6.3. Let $(\mathrm{X}, \mathrm{T})$ and $\left(\mathrm{Y}, \mathrm{T}^{\prime}\right)$ be two Chang's fuzzy topological space and let $f: X \rightarrow Y$ be a mapping. Then $f:(X, T) \rightarrow\left(Y, T^{\prime}\right)$ is continuous if and only if $f:\left(X, T^{[\lambda, \mu]}\right) \rightarrow\left(Y,\left(T^{\prime}\right)^{[\lambda, \mu]}\right)$ is an IVGPmapping, for each $[\lambda, \mu] \in D\left(I_{0}\right)$.

Proof. $(\Rightarrow)$ : Suppose $f:(X, T) \rightarrow\left(Y, T^{\prime}\right)$ is continuous, let $B \in I^{Y}$ and let $[\lambda, \mu] \in D\left(I_{0}\right)$. Then we have the following cases:

(i) $B=\phi$ or $Y$,

(ii) $B \in T^{\prime}$,

(iii) $B \notin T^{\prime}$.

Case (i) : $f^{-1}(\phi)=\phi$ or $f^{-1}(Y)=X$. Thus

$$
\left(T^{\prime}\right)^{[\lambda, \mu]}(B) \leq T^{[\lambda, \mu]}\left(f^{-1}(B)\right) \text {. }
$$

Case (ii) : Clearly $\left(T^{\prime}\right)^{[\lambda, \mu]}(B)=[\lambda, \mu]$. Since $f$ is continuous, $f^{-1}(B) \in$ $T$. Thus $T^{[\lambda, \mu]}\left(f^{-1}(B)\right)=[\lambda, \mu]$. So

$$
\left(T^{\prime}\right)^{[\lambda, \mu]} \leq T^{\lambda, \mu}\left(f^{-1}(B)\right) \text {. }
$$

Case(iii) : It is clear that $\left(T^{\prime}\right)^{\lambda, \mu}(B)=\mathbf{0}$. Thus

$$
\mathbf{0}=\left(T^{\prime}\right)^{[\lambda, \mu]}(B) \leq T^{\lambda, \mu}\left(f^{-1}(B)\right) .
$$

So, in all cases, $f:\left(X, T^{\lambda, \mu}\right) \rightarrow\left(Y,\left(T^{\prime}\right)^{[\lambda, \mu]}\right)$ is an IVGP-mapping.

$(\Leftarrow)$ : It follows from Proposition 3.18 and Theorem 6.2.

The following is the immediate result of Definition 6.1. 
Proposition 6.4. Let $(X, \tau),(Y, \eta)$ and $(Z, \xi)$ be IVSTSs.

(a) $1_{X}:(X, \tau) \rightarrow(X, \tau)$ is an IVGP-mapping.

(b) If $f:(X, \tau) \rightarrow(Y, \eta)$ and $g:(Y, \eta) \rightarrow(Z, \xi)$ is IVGP-mappings, then $g \circ f:(X, \tau) \rightarrow(Z, \xi)$ is an IVGP-mapping.

We can easily see that the collection of all IVFTSs and IVGP-mapping between then forms a concrete category and we will denote it by IVTop.

Theorem 6.5. Let $(X, \tau)$ be an IVFTS and let $f: X \rightarrow Y$ be a mapping. Let $\left\{T_{\lambda, \mu}\right\}_{\lambda, \mu} \in D\left(I_{0}\right)$ be a descending family of chang's fuzzy topologies on $Y$. Let $\eta$ be the IVGO on $X$ generated by this family. For each $[\lambda, \mu] \in D\left(I_{0}\right)$, suppose $\mathfrak{B}_{[\lambda, \mu]}$ or $\mathcal{S}_{[\lambda, \mu]}$ is a base or a subbase for $T_{[\lambda, \mu]}$, respectively. Then the followings are equivalent:
(a) $f:(X, \tau) \rightarrow(y, \eta)$ is an IVGP-mapping.
(b) $\tau\left(f^{-1}(B)\right) \geq[\lambda, \mu], \forall B \in T_{[\lambda, \mu]}, \forall[\lambda, \mu] \in D\left(I_{0}\right)$.
(c) $\tau\left(f^{-1}(B)\right) \geq[\lambda, \mu], \forall B \in \mathfrak{B}_{[\lambda, \mu]}, \forall[\lambda, \mu] \in D\left(I_{0}\right)$.
(d) $\tau\left(f^{-1}(B)\right) \geq[\lambda, \mu], \forall B \in \mathcal{S}_{[\lambda, \mu]}, \forall[\lambda, \mu] \in D\left(I_{0}\right)$.

Proof. $(a)(\Rightarrow)(b)$ : Suppose (a) holds. Let $[\lambda, \mu] \in D\left(I_{0}\right)$ and let $B \in T_{[\lambda, \mu]}$.

Then $\tau\left(f^{-1}(B)\right) \geq \zeta(B) \geq[\lambda, \mu]$.

It is obvious that (b) $\Rightarrow$ (c) $\Rightarrow$ (d) hold.

$(d)(\Rightarrow)(a)$ : Suppose (d) holds. Let $B \in I^{Y}$ and, without loss of generality, let $\eta(B)=[\lambda, \mu]>\mathbf{0}$. Then $B \in T_{[\lambda, \mu]}$. Now, $\mathrm{B}$ is of the form, $B=\bigcup_{\alpha \in \Gamma} B_{\alpha}$, where $B_{\alpha} \in \mathfrak{B}_{[\lambda, \mu]}, \forall \alpha \in \Gamma$. Also, for each $\alpha \in \Gamma, B_{\alpha}$ is of the form, $B_{\alpha}=\bigcup_{j=1}^{n_{\alpha}} S_{\alpha, j}$, where $S_{\alpha, j} \in \mathcal{S}_{[\lambda, \mu]}, \forall j=1,2, \cdots, n_{\alpha}$. Thus

$$
\begin{aligned}
& \tau\left(f^{-1}(B)\right)=\tau\left(f^{-1}\left(\bigcup_{\alpha \in \Gamma}\left(\bigcap_{j=1}^{n_{\alpha}} S_{\alpha, j}\right)\right)\right) \\
& =\tau\left(\bigcup_{\alpha \in \Gamma}\left(\bigcap_{j=1}^{n_{\alpha}} f^{-1}\left(S_{\alpha, j}\right)\right)\right) \\
& \left.\geq \bigwedge_{\alpha \in \Gamma}\left(\bigwedge_{j=1}^{n_{\alpha}} \tau f^{-1}\left(S_{\alpha, j}\right)\right)\right)[\text { Since } \tau \in \operatorname{IVGO}(\mathrm{X})] \\
& \geq[\lambda, \mu] .[\text { By the condition }(\mathrm{d})]
\end{aligned}
$$

So $\tau\left(f^{-1}(B)\right) \geq \eta(B)$. Hence $f:(X, \tau) \rightarrow(Y, \eta)$ is on IVGP-mapping. This completes the proof. 


\section{References}

[1] K. Atanassov, Intuitionistic fuzzy sets, Fuzzy Sets and Systems 20(1) (1986) 87-96.

[2] K. Atanassov, New operators defined over the intuitionistic fuzzy sets, Fuzzy Sets and Systems 61(2)(1993) 131-142.

[3] K. Atanassov and G. Gargov, Interval-valued intuitionistic fuzzy sets, Fuzzy Sets and Systems 31(3)(1989) 343-349.

[4] P. Burillo and H. Bustince, Two operators on interval-valued intuitionistic fuzzy sets: Part II, C.R. Acad. Bulg. Sci. 48(1)(1995) 17-20.

[5] H. Bustince and P. Burillo, Correlation of interval-valued intuitionistic fuzzy sets, Fuzzy Sets and Systems 74(1995) 237-244.

[6] C.L. Chang, Fuzzy topological spaces, J. Math. Anal. Appl. 24(1968) 182-190.

[7] K.C. Chattopadhyay, R.N. Hazra and S.K. Samanta, Gradation of openness:fuzzy topology, Fuzzy Sets and Systems 49(1992) 237-242.

[8] D.Çoker, An introduction to intuitionistic fuzzy topological spaces, Fuzzy Sets and Systems 88(1997) 81-89.

[9] V. Gregori and A.Vidal, Gradation of openness and Chang's fuzzy topologies, Fuzzy Sets and systems 109(2000) 233-244.

[10] R.N. Hazra and S.K. Samanta, K.C. Chattopadhyay, Fuzzy topology redefined, Fuzzy Sets and Systems 45(1992)79-82.

[11] K.Hur, Y.B.Kim and J.H.Ryou, Intuitionistic fuzzy topological groups, Honam Math. J. 26(2)(2004) 163-192.

[12] K.Hur, J.H.Kim and J.H.Ryou, Intuitionistic fuzzy topological spaces, J.Korea Soc. Math. Educ. Ser. B: Pure Appl. Math. 11(3)(2004) 243265.

[13] R. Lowen, Fuzzy topological spaces and fuzzy compactness, J. Math. Anal. Appl. 56(1976), 621-623.

[14] T.K.Modal and S.K.Samanta, On intuitionistic gradation of openness, Fuzzy Sets and Systems 131(2002) 323-336.

[15] S.K. Samanta and T.K. Mondal, Intuitionistic gradation of openness: intuitionistic fuzzy topology, Busefal 73(1997) 8-17.

[16] , Topology of interval-valued fuzzy sets, Indian J. Pure Appl. Math. 30(1)(1999) 23-38.

[17] _, Topology of interval-valued intuitionistic fuzzy sets, Fuzzy Sets and Systems 119(2001) 483-494.

[18] A. Šostak, On a fuzzy topological structure, Rend. Circ. Mat. Palermo:Suppl. Ser. II(1985) 89-103.

[19] L.A. Zadeh, Fuzzy sets, Inform. and Control 8(1965) 338-353. 
$[20]_{\text {_ }}$, The concept of a linguistic variable and its application to approximate reasoning I, Inform. Sci. 8(1975) 199-249.

${ }^{1}$ Jeong Yeol Choi

Professor of Wonkwang University

Research Area : Fuzzy Set Theory, Analysis

E-mail : jychoi@wonkwang.ac.kr

${ }^{2}$ So Ra Kim

Graduate Student

Research Area: Fuzzy Topology, Fuzzy Algebra, Dynamic Theory

E-mail : soraking@naver.com

${ }^{3} \mathrm{Kul} \mathrm{Hur}$

Professor of Wonkwang University

Research Area: Fuzzy Topology, Fuzzy Algebra, Hyperspaces, Category

Theory E-mail : kulhur@wonkwang.ac.kr

${ }^{1,2,3}$ Division of Mathematics and Informational Statistics, and Nanoscale Science and Technology Institute, Wonkwang University,

Iksan, Chonbuk, Korea 570-749 